\title{
A plasma-derived extracellular vesicle mRNA classifier for the detection of breast cancer
}

\author{
Geng-Xi Cai ${ }^{1,2 \#} \wedge$, Li Lin ${ }^{3 *}$, Xiang-Ming Zhai ${ }^{3 \#}$, Zhi-Wei Guo ${ }^{3}$, Ying-Song Wu ${ }^{3}$, Guo-Lin Ye $^{2}$, Qing Liu ${ }^{2}$, \\ Lu-Shi Chen ${ }^{2}$, Guan-Yu Xing ${ }^{2}$, Qiao-Hong Zhao ${ }^{2}$, Li-Ling Tang ${ }^{2}$, Shun-He Mai ${ }^{2}$, Bo-Jian Ye $^{2}$ \\ ${ }^{1}$ Breast Tumor Center, Sun Yat-Sen Memorial Hospital, Sun Yat-Sen University, Guangzhou, China; ${ }^{2}$ Department of Breast Surgery, The First \\ People's Hospital of Foshan, Foshan, China; ${ }^{3}$ Key Laboratory of Antibody Engineering of Guangdong Higher Education Institutes, School of \\ Laboratory Medical and Biotechnology, Southern Medical University, Guangzhou, China \\ Contributions: (I) Conception and design: GX Cai; (II) Administrative support: GX Cai; (III) Provision of study materials or patients: GL Ye, Q Liu, \\ LS Chen, GY Xing, QH Zhao, LL Tang, SH Mai, BJ Ye; (IV) Collection and assembly of data: Q Liu, LS Chen, GY Xing, QH Zhao, LL Tang, SH \\ Mai, BJ Ye; (V) Data analysis and interpretation: L Lin, XM Zhai, GL Ye, Q Liu, LS Chen, GY Xing, QH Zhao, LL Tang, SH Mai, BJ Ye, ZW Guo, \\ YS Wu; (VI) Manuscript writing: All authors; (VII) Final approval of manuscript: All authors. \\ \#These authors contributed equally to this work. \\ Correspondence to: Geng-Xi Cai. Sun Yat-Sen Memorial Hospital, Sun Yat-Sen University, 107 West Yanjiang Road, Guangzhou 510120, China; The \\ First People's Hospital of Foshan, 81 North Lingnan Avenue, Foshan 528000, China. Email: caigengxi2021@126.com.
}

Backgroundk According to the global cancer burden data released in 2020, breast cancer (BC) has become the most common cancer in the world. Similar to those of other cancers, the present methods used in clinic for diagnosing early BC are invasive, inaccurate, and insensitive. Hence, new non-invasive methods capable of early diagnosis are needed.

Methods: We applied next-generation sequencing and analyzed the messenger RNA (mRNA) profiles of plasma extracellular vesicles (EVs) derived from 14 BC patients and 6 patients with benign breast lesions. We used 3 regression models, namely support vector machine (SVM), linear discriminate analysis (LDA), and logistic regression (LR), to develop classifiers for use in making predictive BC diagnoses; and used 259 plasma samples, including those obtained from 144 patients with BC, 72 patients with benign breast lesions, and 43 healthy women, which were divided into training groups and validation groups to verify their performances as classifiers by quantitative reverse transcription polymerase chain reaction (RT-qPCR). The area under the curve (AUC) and accuracy, sensitivity, and specificity of the classifiers were cross-validated with the leave-1-out cross-validation (LOOCV) method.

Results: Among all combinations assessed with the 3 different regression models, an 8-mRNA combination, named $\mathrm{EXOB}_{\mathrm{mRNA}}$, exhibited high performance [accuracy $=71.9 \%$ and $\mathrm{AUC}=0.718,95 \%$ confidence interval (CI): 0.652 to 0.784 ] in the training cohort after LOOCV was performed, showing the largest AUC in the SVM model. The mRNAs in $\mathrm{EXOB}_{\mathrm{mRNA}}$ were HLA-DRB1, HAVCR1, ENPEP, TIMP1,

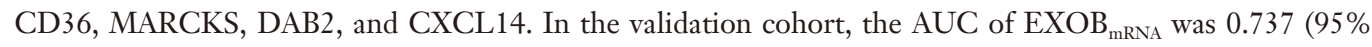
CI: 0.636 to 0.837$)$. In addition, gene function and pathway analyses revealed that different levels of gene expression were associated with cancer.

Conclusions: We developed a high-performing predictive classifiers including $8 \mathrm{mRNAs}$ from plasma extracellular vesicles for diagnosing breast cancer.

Keywords: Breast cancer (BC); messenger RNAs (mRNAs); extracellular vesicles (EVs); diagnosis; predictive classifier

Submitted Apr 02, 2021. Accepted for publication Jun 11, 2021.

doi: $10.21037 /$ gs-21-275

View this article at: https://dx.doi.org/10.21037/gs-21-275

\footnotetext{
$\wedge$ ORCID: 0000-0003-0575-4314.
} 


\section{Introduction}

According to the latest global cancer burden data released by the World Health Organization (WHO) in 2020, the number of new cases of breast cancer (BC) has increased rapidly, replacing lung cancer as the most common cancer, and comprising $11.7 \%$ of all cancer cases. Among women, $\mathrm{BC}$ is the most frequently diagnosed cancer, accounting for approximately $25 \%$ of cancer cases and is the main cause of cancer death (1). Similar to other types of cancer, early diagnosis can reduce the incidence and mortality rates of $\mathrm{BC}$, and is the key to improving the survival rate. Therefore, early diagnosis of $\mathrm{BC}$ has become an important aspect in the treatment of $\mathrm{BC}$.

In various platforms, imaging techniques are major diagnostic methods and include ultrasound scanning, mammography, magnetic resonance imaging (MRI), and positron-emission tomography (PET), which can provide valuable data for the treatment of $\mathrm{BC}$ patients. However, some of these techniques are expensive, lack sensitivity for detecting early $\mathrm{BC}$, and/or expose the patient to radiation, which adds an extra risk factor. In addition, some of the most studied and established BC biomarkers, such as CEA, CA125, and CA15-3, have been used to detect and monitor the diagnosis of $\mathrm{BC}$ in patients; however, low sensitivity limits their applications in early BC detection (2). Moreover, the currently available diagnostic technologies for use with $\mathrm{BC}$ have certain limitations, and the probability of falsepositive results is $20-50 \%$ (3). Therefore, there is an urgent need for non-invasive methods that can more sensitively and specifically identify BC in the early stage.

Extracellular vesicles (EVs) produced by cells are mainly divided into three types according to their size and biogenesis: exosomes (40-200 nm), microvesicles (200-1,000 nm) and apoptotic bodies (500-2,000 nm) (4). EVs play an important role in breast physiology and pathophysiology including carcinogenesis, tumor progression, molding the tumor microenvironment, and immune modulation. Exosomes, as a class of EVs, are nano-vesicles related to the physiologic regulation of breast development (such as lactation), but they are also significant mediators of breast tumors, endowing them with great potential as new molecular markers for the diagnosis and prognosis of BC (5). Exosomes are considered to be important mediators of cellto-cell communication, through the horizontal transfer of bioactive substances, such as proteins (6), messenger RNA (mRNA) and non-coding RNA (7). Considerable evidence has suggested that mRNAs enriched in serum exosomes can be effectively internalized recipient cells $(8,9)$. Therefore, the mRNA in exosomes is a potential biomarker for use in liquid biopsy, and the use of exosomal profiling without tissue has obvious prospects for the early diagnosis of diseases. Up to now, there is no research on developing BC diagnosis model by detecting multiple plasma-derived $\mathrm{EV}$ mRNAs.

In this study, we exploited a non-invasive method for the diagnosis of BC. First, we employed next-generation sequencing to determine the RNA profiles of plasma EVs derived from 14 patients with $\mathrm{BC}$ and 6 patients with benign breast lesions serving as controls. Next, we compared these RNA profiles to identify the mRNAs of plasma EVs at significantly different levels. Finally, we developed predictive classifiers with multiple machine learning models to discriminate patients with $\mathrm{BC}$ from individuals without cancer. In summary, our study may provide some new suggestions for the diagnosis of BC.

We present the following article in accordance with the STARD reporting checklist (available at https://dx.doi. org/10.21037/gs-21-275).

\section{Methods}

\section{Sample characteristics}

A total of 259 plasma samples were derived from 3 groups of participants enrolled at The First People's Hospital of Foshan, including 144 with BC, 72 benign with breast lesions, and 43 healthy women. All the plasma samples of cancer patients were prospectively collected during the discovery stage and before cancer therapy was initiated. The plasma samples of the discovery cohort were prospectively collected between January 2018 and July 2018. The samples comprising the training and validation cohorts were prospectively collected between August 2018 and May 2019. With the informed consent of all participants, all plasma samples were collected for research according to the protocols approved by the Ethics Committee of the First People's Hospital of Foshan (ID: L[2021]-7). All procedures performed in this study involving human participants were in accordance with the Declaration of Helsinki (as revised in 2013). Details regarding the clinical information of the participants involved in this study are listed in Table S1.

\section{$R N A$ isolation from Plasma $E V s$}

Total RNA was isolated using an exoRNeasy Serum/Plasma 
Maxi Kit (Qiagen, Valencia, CA, USA) according to the manufacturer's protocol. We purified exosomes and other EVs and isolated total versicular RNA, including mRNA, non-coding RNA, miRNA and other small RNAs, from 2-4 mL serum. In brief, the plasma was prefiltered, then was mix with $2 \times$ binding buffer. The mixture is added to the exoEasy membrane affinity to bind the EVs to the membrane. After centrifugation, the wash buffer was added to wash off non-specific material in the column. After enriching EVs, QIAZOL was added to the column to lyse the vesicles and chloroform was added to the lysate collected after centrifugation. The aqueous phases os recovered and mixed with ethanol, and the sample-ethanol mixture is added to the RNeasy MinElute spin column and centrifuged. Wash the column with buffer RWT, then wash twice with buffer RPE. And finally elute RNA in water.

\section{Library preparation and next-generation sequencing}

A high-throughput sequencing service, transcriptome highthroughput sequencing, and subsequent bioinformatics analysis were provided by Cloud-Seq Biotech (Shanghai, China). Briefly, total RNA was used to remove ribosomal RNA (rRNA) prior to the construction of the RNA-seq libraries with Ribo-Zero rRNA Removal Kits (Illumina, San Diego, CA, USA) following the manufacturer's instructions. The RNA libraries were prepared using a TruSeq Stranded Total RNA Library Prep Kit (Illumina) with rRNAdepleted RNAs following the manufacturer's instructions. A BioAnalyzer 2100 system (Agilent Technologies, Santa Clara, CA, USA) was used to quantify and control the quality of the libraries. According to the manufacturer's instructions, $10 \mathrm{pM}$ libraries were denatured as singlestranded DNA molecules, captured in Illumina flow cells, and amplified into clusters in situ. Finally, each nucleic acid sequence was sequenced for 150 cycles on an Illumina HiSeq Sequencer.

\section{Data analysis of RNA-sequencing}

Paired-end reads were obtained from an Illumina HiSeq 4000 sequencer, and quality control was performed by Q30. Low-quality reads were filtered by Cutadapt software (v1.9.3) after 3' adaptor trimming (9). The high-quality trimmed reads were used to analyze mRNAs and were aligned to the human reference genome (UCSC hg19) using HISAT2 software v2.0.4 (http://www.ccb.jhu.edu/software/ hisat/index.shtml) (10). The FPKM was obtained by using
Cuffdiff software (v2.2.1, in the Cufflinks package) (10) and used for the mRNA expression profiles as guided by the Ensembl gtf gene annotation file. Then, fold change and $\mathrm{P}$ value were determined based on the FPKM to identify the differentially expressed mRNAs. We performed GO and pathway enrichment analyses with the clusterProfiler package on the mRNAs expressed at differential levels.

\section{Quantitative real-time polymerase chain reaction}

Complementary DNA was synthesized using a PrimeScript RT reagent Kit (Takara, Bio Inc., Kusatsu, Shiga, Japan) after RNA isolation. mRNA quantification was obtained with qPCR using SYBR Premix Ex Taq (Takara, Bio Inc.) and an ABI7500 Real-Time PCR system (Applied Biosystems, Forster City, CA, USA) with the primers and complementary DNA (cDNA) template recommended by the manufacturer. The primers are listed in Table S2.

\section{Classifier construction}

To develop classifiers for $\mathrm{BC}$ diagnosis, 13 candidate mRNAs with significantly elevated levels in BC patients were selected and assessed using quantitative real-time polymerase chain reaction (qRT-PCR). A total of 259 participants were divided into training and validation cohorts at a ratio of 7:3. The training cohort contained 182 participants, including 101 patients with $\mathrm{BC}$ and 81 without cancer serving as controls (51 individuals with benign breast lesions and 30 healthy women). The validation cohort contained 77 participants, including 43 patients with BC and 34 without cancer serving as controls (21 individuals with benign breast lesions and 13 healthy women). To construct predictive classifiers that can be used to differentiate individuals with BC from those serving as controls, we used 3 regression models: support vector machine (SVM), logistic regression (LR), and linear discriminate analysis (LDA). As numerous studies have reported that discrete data may improve classifier performance (11), continuous variables were discretized before classifier construction according to the optimal cut-off point, which was selected according to the maximum value of (sensitivity + specificity) $/ 2$ in the training cohort. Then, the continuous value was set to 1 when it was higher than the corresponding optimal cutoff for each subject; otherwise, it was set to 0 (Table S3). Using the stepwise method, the optimal classifier with the largest AUC was selected. The leave-1-out cross-validation (LOOCV) method was used to estimate the robustness and 
prediction error of the selected classifiers. In the validation cohort, the relative levels of the mRNAs in the selected classifiers were determined, and the predictive efficacy of the classifiers was evaluated in the test cohort.

\section{Statistical analysis}

We used R software (version 3.4; https://www.R-project. org/) for statistical analyses. Mann-Whitney U test or Kruskal-Wallis test was used to compare the differences in patient characteristics in 2 or more groups, respectively, and Pearson chi-square was used to compare sample compositions. The diagnostic accuracy of the candidate mRNAs or their combinations was evaluated by receiver operating characteristic (ROC) curve analysis with the area under the ROC curve (AUC). A P value $<0.05$ was considered significant.

\section{Results}

\section{Significantly differential mRNAs identification}

To identify different levels of plasma EV mRNAs between patients with $\mathrm{BC}$ and those without cancer, we first generated the mRNA profiles of the exosomes obtained from the plasma of 14 participants with $\mathrm{BC}$ and 6 with benign lesions during the discovery stage. We found that the levels of 1,156 mRNAs were significantly different, including 183 mRNAs at higher levels and 973 mRNAs at lower levels (Figure 1A, Table S4, $\mid \log 2$-fold changel $>2, \mathrm{P}$ value $<0.05)$. By implementing unsupervised clustering analysis, we found distinct patterns between the participants with and without cancer, and the samples in each group clustered together in separate groups (Figure 1B), which indicated the potential of using mRNAs from EVs for screening BC.

\section{Gene function and pathway enrichment analyses}

To reveal the potential roles of mRNAs as indicated by their different levels in cases of tumorigenesis, we performed a gene function enrichment analysis. The results indicated that the terms associated with these mRNAs were enriched in multiple processes, such as multiple biological pathways and processes related to immunity (Figure 1C). For example, HCK participates in a variety of biological processes by mediating immune response regulation signaling pathways and multiple signaling pathways [including epithelial to mesenchymal transition (EMT), the PI3K/AKT signaling pathway, and focal adhesion] in BC (12). In addition, AXL is involved in a variety of processes that promote tumor formation and metastasis, and is considered an effective biomarker for BC. Especially in triple negative (TN)BC tumors, AXL is considered a key factor in promoting EMT associated with mesenchymal and invasive phenotypes (13). In addition, we performed a pathway enrichment analysis and found that some of the mRNAs in BC are closely related to ribosomes (Figure 1D). These mRNAs may play important roles in the translation process.

\section{Classifier construction}

For clinical practice and detection convenience, we focused on the mRNAs of plasma EVs with elevated levels. According to fold change, the false discovery rate (FDR) value and mean levels, we selected 12 mRNAs for further qRT-PCR identification and predictive classifier construction. To develop predictive classifiers for BC diagnosis, the samples were split into training and validation cohorts. Using qRT-PCR, we assessed the relative levels of 12 mRNAs in the training group that were at higher levels in the 182 plasma samples obtained from $101 \mathrm{BC}$ participants and 81 patients without cancer serving as controls (51 participants with benign breast lesions and 30 healthy women). We used 3 regression models, SVM, LDA, and LR, to construct mRNA classifiers that could be used to differentiate individuals with $\mathrm{BC}$ from those without cancer serving as controls. The AUC and accuracy, sensitivity, and specificity of the classifiers were cross-validated with the LOOCV method (Figure 2). Among all combinations assessed with the 3 different regression models, an 8-mRNA combination, named $\mathrm{EXOB}_{\mathrm{mRNA}}$, achieved high performance [AUC $=0.718,95 \%$ confidence interval (CI): 0.652 to 0.784 , and accuracy $=71.9 \%$ ] in the training cohort after LOOCV was performed, showing the largest AUC with the SVM model (Figure $2 A$ ). The mRNAs in $\mathrm{EXOB}_{\mathrm{mRNA}}$ were HLA-DRB1, HAVCR1, ENPEP, TIMP1, CD36, MARCKS, DAB2, and CXCL14. Then, the performance of $\mathrm{EXOB}_{\mathrm{mRNA}}$ was subsequently studied and verified in the validation cohort. In the validation cohort, the AUC for the $\mathrm{EXOB}_{\mathrm{mRNA}}$ group was 0.737 (95\% CI: 0.636 to 0.837 , Figure $2 E, F)$. In contrast with that of the training cohort, the AUC of the validation cohort was similar to that of the training cohort $(\mathrm{P}=0.764)$.

\section{Discussion}

Our study found 1,156 mRNAs at different levels between 


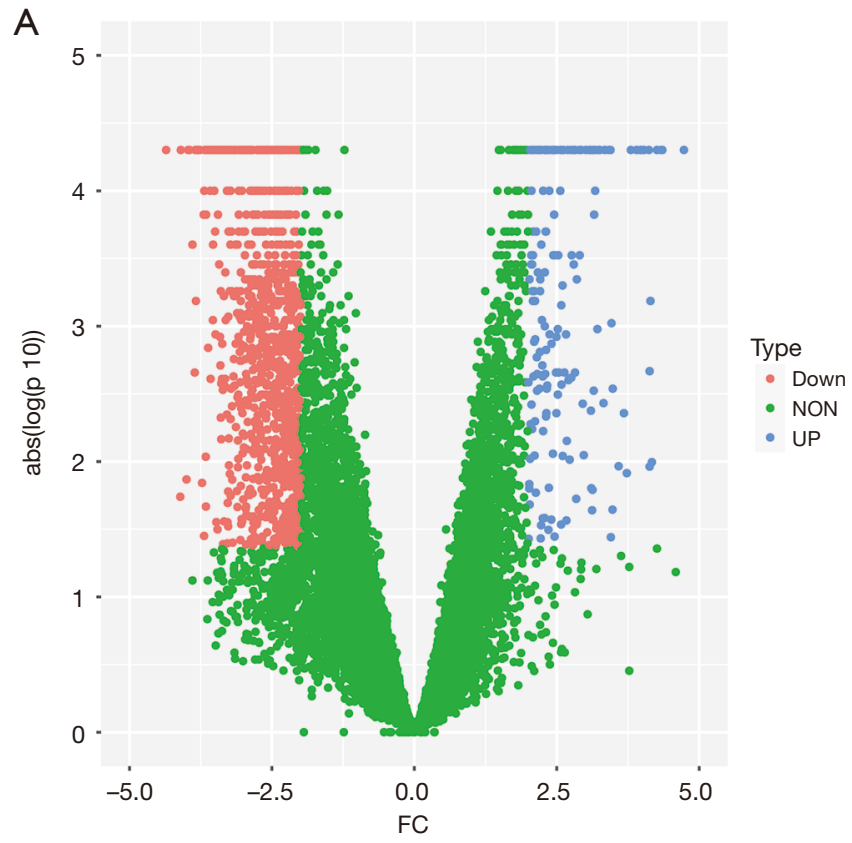

B

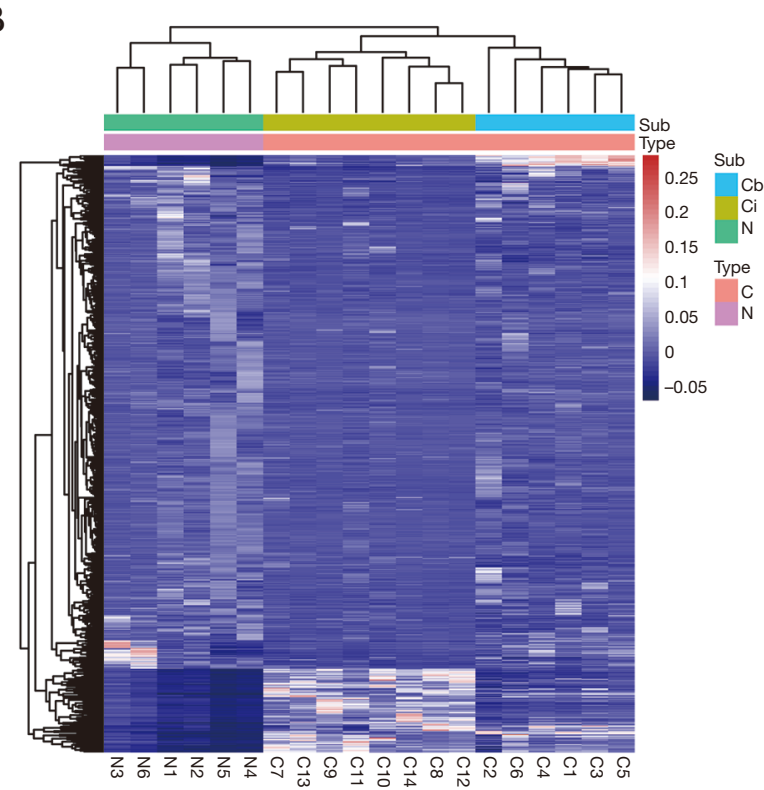

C

Biological process
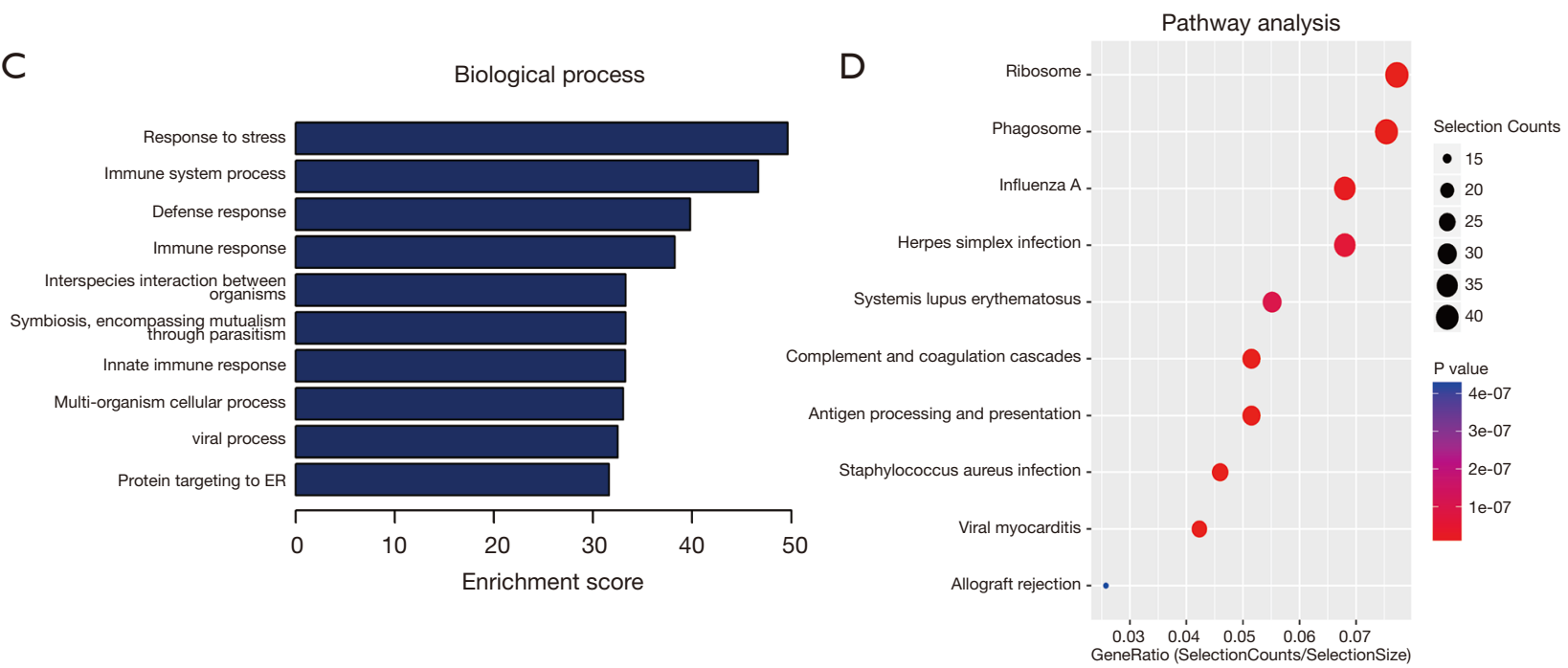

Figure $1 \mathrm{mRNA}$ with differential levels between breast cancer and non-cancer controls. (A) Volcano plots of the mRNA profiles of plasma EVs derived from 14 patients with BC and 6 without cancer $\left(\mid \log _{2}\right.$ fold change $\mid \geq 2$ and FDR $\left.\leq 0.2\right)$. (B) Heat map of the mRNAs with differential read coverages. (C) Top 10 enriched GO biological processes. (D) Top 10 enriched pathway analysis. EVs, extracellular vesicles; mRNA, messenger RNA; BC, breast cancer; GO, Gene Ontology; FDR, false discovery rate.

14 participants with $\mathrm{BC}$ and 6 participants with benign breast lesions by comparing the mRNA profiles using next-generation sequencing. In addition, gene function and pathway analyses showed that the genes expressed at differential levels were related to cancer. Finally, we developed predictive classifiers to assess their potential for $\mathrm{BC}$ diagnosis, and we found that the $\mathrm{AUC}$ of the $\mathrm{EXOB}_{\mathrm{mRNA}}$ group was 0.724 (0.669 to 0.779$)$.

Our method is non-invasive, and detection is not confounded by the heterogeneity of a tumor. At the same time, it does not expose patients to the effects of radiation and shows high sensitivity and specificity, highlighting great potential for use in the early screening of BC. However, this method has certain limitations. The cost of extracting 
A

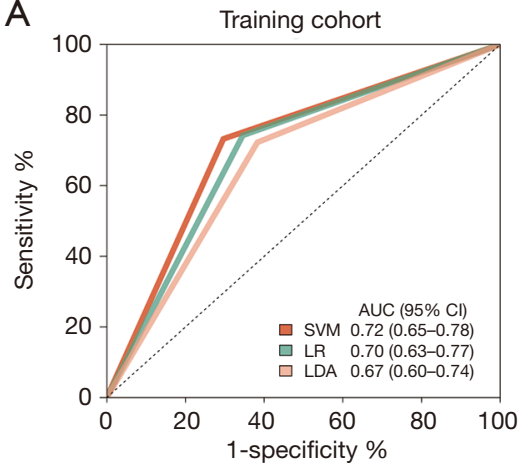

B

\begin{tabular}{ccccc}
\multicolumn{5}{c}{ Training cohort } \\
\hline Model & Acc & Sen & Spe & P \\
\hline SVM & 72.0 & 73.3 & 70.4 & \\
LR & 70.3 & 74.3 & 65.4 & 0.3501 \\
LDA & 67.6 & 72.3 & 61.7 & 0.0307 \\
\hline
\end{tabular}

C

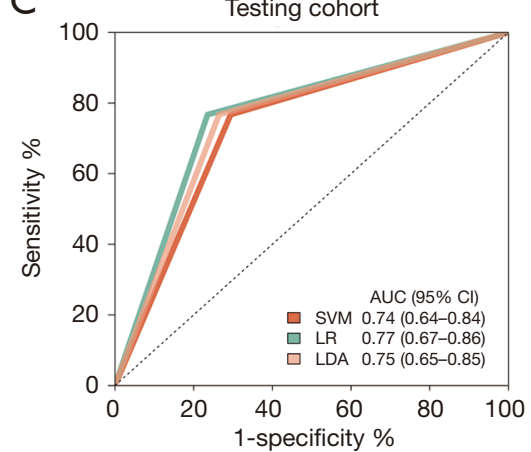

D

\begin{tabular}{ccccc}
\multicolumn{5}{c}{ Testing cohort } \\
\hline Model & Acc & Sen & Spe & P \\
\hline SVM & 74.0 & 76.7 & 70.6 & \\
LR & 76.6 & 76.7 & 76.5 & 0.151 \\
LDA & 75.3 & 76.7 & 73.5 & 0.3173 \\
\hline
\end{tabular}

E

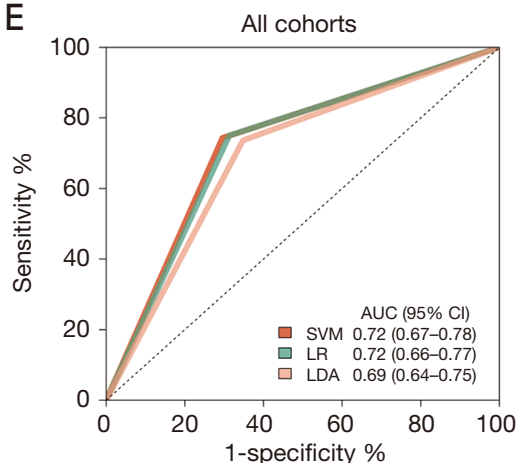

$\mathrm{F}$

\begin{tabular}{ccccc}
\multicolumn{5}{c}{ All cohorts } \\
\hline Model & Acc & Sen & Spe & P \\
\hline SVM & 72.6 & 74.3 & 70.4 \\
LR & 72.2 & 75.0 & 68.7 & 0.7465 \\
LDA & 69.9 & 73.6 & 65.2 & 0.0718 \\
\hline
\end{tabular}

Figure 2 The performance of predictive classifiers. The roc curves of the predictive classifiers in the training (A), testing (C), and all (E) cohorts. The predictive efficacy of the classifiers the training (B), testing (D), and all (F) cohorts. The performance of classifiers in training cohort was cross-validated using LOOCV. $\mathrm{P}=$ the $\mathrm{P}$ value of the comparison of 2 AUCs. LOOCV, leave-1-out cross-validation; ROC, receiver operating characteristic; Acc, accuracy; Sen, sensitivity; Spe, specificity; AUC, area under the curve.

exosomes is still very high, kits are expensive, and an ultracentrifuge is often lacking in the clinic; therefore, this method is still difficult to use extensively in the clinic. In addition, the classifier model needs to be further optimized, and the biological function of each mRNA and the relationship between them need to be further studied. With optimization and further characterization, the possibility that this method will be used in clinical applications will be increased.

We searched the literature for the genes that we identified and discovered that many of the mRNAs and their translated proteins are closely related to BC (14). For instance, DAB2 can be used as a novel marker and therapeutic target for BC (15), MARCKS is closely related to poor prognosis of $\mathrm{BC}$, and knockdown of ENPEP can inhibit the invasion of BC (16). In addition, there have been many research reports on these genes in other tumors. First, they can be used as tumor risk genes or prognostic genes, and their expression levels in tumor patients are significantly increased. For example, HLA-DRB1 is considered a risk gene for cervical cancer (17), and TIMP1 may be related to the metastasis of uveal melanoma (UM), possibly playing an important role in identifying patients with high metastatic risk and predicting the prognosis of UM (18). Second, these genes are important for the occurrence and development of tumors. For instance, CD36 upregulates DEK transcription and promotes cell migration and invasion and EMT in gastric cancer (19). Overexpression of HAVCR1 can reduce cell adhesion and invasion in colorectal cancer (8). Finally, these genes can play important roles in tumor immunity and the tumor microenvironment and are potential targets for future immunotherapy. For instance, the chemokine CXCL14 is often dysregulated in several types of cancer, and its destructive effect limits key antitumor immune regulator function and is closely related to poor patient prognosis.

Therefore, we expect that the biological functions and characteristics of these mRNAs can be combined to construct different classifiers for use in early tumor diagnosis or subtype classification of tumors.

\section{Acknowledgments}

Funding: The work was supported by the Medical Scientific Research Foundation of Guangdong Province of China (B2017006); Special fund of Foshan Summit plan (2020G010); and Foshan City medical science and technology project (2020001005030). 


\section{Footnote}

Reporting Checklist: The authors have completed the STARD reporting checklist. Available at https://dx.doi. org/10.21037/gs-21-275

Data Sharing Statement: Available at https://dx.doi. org/10.21037/gs-21-275

Conflicts of Interest: All authors have completed the ICMJE uniform disclosure form (available at https://dx.doi. org/10.21037/gs-21-275). The authors have no conflicts of interest to declare.

Ethical Statement: The authors are accountable for all aspects of the work in ensuring that questions related to the accuracy or integrity of any part of the work are appropriately investigated and resolved. With the informed consent of all participants, all plasma samples were collected for research according to the protocols approved by the Ethics Committee of the First People's Hospital of Foshan (ID: L[2021]-7). All procedures performed in this study involving human participants were in accordance with the Declaration of Helsinki (as revised in 2013).

Open Access Statement: This is an Open Access article distributed in accordance with the Creative Commons Attribution-NonCommercial-NoDerivs 4.0 International License (CC BY-NC-ND 4.0), which permits the noncommercial replication and distribution of the article with the strict proviso that no changes or edits are made and the original work is properly cited (including links to both the formal publication through the relevant DOI and the license). See: https://creativecommons.org/licenses/by-nc-nd/4.0/.

\section{References}

1. Sung H, Ferlay J, Siegel RL, et al. Global Cancer Statistics 2020: GLOBOCAN Estimates of Incidence and Mortality Worldwide for 36 Cancers in 185 Countries. CA Cancer J Clin 2021;71:209-49.

2. Jafari SH, Saadatpour Z, Salmaninejad A, et al. Breast cancer diagnosis: Imaging techniques and biochemical markers. J Cell Physiol 2018;233:5200-13.

3. Lindenberg MA, Miquel-Cases A, Retèl VP, et al. Imaging performance in guiding response to neoadjuvant therapy according to breast cancer subtypes: A systematic literature review. Crit Rev Oncol Hematol 2017;112:198-207.
4. Kim KM, Abdelmohsen K, Mustapic M, et al. RNA in extracellular vesicles. Wiley Interdiscip Rev RNA 2017;8:10.1002/wrna.1413.

5. Zha QB, Yao YF, Ren ZJ, et al. Extracellular vesicles: An overview of biogenesis, function, and role in breast cancer. Tumour Biol 2017;39:1010428317691182.

6. Théry C, Zitvogel L, Amigorena S. Exosomes: composition, biogenesis and function. Nat Rev Immunol 2002;2:569-79.

7. Valadi H, Ekström K, Bossios A, et al. Exosome-mediated transfer of mRNAs and microRNAs is a novel mechanism of genetic exchange between cells. Nat Cell Biol 2007;9:654-9.

8. Wang Y, Martin TA, Jiang WG. HAVcR-1 expression in human colorectal cancer and its effects on colorectal cancer cells in vitro. Anticancer Res 2013;33:207-14.

9. Mitsuhashi M, Taub DD, Kapogiannis D, et al. Aging enhances release of exosomal cytokine mRNAs by A $\beta 1-42$ stimulated macrophages. FASEB J 2013;27:5141-50.

10. Trapnell C, Roberts A, Goff L, et al. Differential gene and transcript expression analysis of RNA-seq experiments with TopHat and Cufflinks. Nat Protoc 2012;7:562-78.

11. Lin XJ, Chong Y, Guo ZW, et al. A serum microRNA classifier for early detection of hepatocellular carcinoma: a multicentre, retrospective, longitudinal biomarker identification study with a nested case-control study. Lancet Oncol 2015;16:804-15.

12. Zhu X, Zhang Y, Bai Y, et al. HCK can serve as novel prognostic biomarker and therapeutic target for Breast Cancer patients. Int J Med Sci 2020;17:2773-89.

13. Falcone I, Conciatori F, Bazzichetto C, et al. AXL Receptor in Breast Cancer: Molecular Involvement and Therapeutic Limitations. Int J Mol Sci 2020;21:8419.

14. Tian X, Zhang Z. miR-191/DAB2 axis regulates the tumorigenicity of estrogen receptor-positive breast cancer. IUBMB Life 2018;70:71-80.

15. Manai M, Abdeljaoued S, Goucha A, et al. MARCKS protein overexpression is associated with poor prognosis in male breast cancer. Cancer Biomark 2019;26:513-22.

16. Feliciano A, Castellvi J, Artero-Castro A, et al. miR-125b acts as a tumor suppressor in breast tumorigenesis via its novel direct targets ENPEP, CK2- $\alpha$, CCNJ, and MEGF9. PLoS One 2013;8:e76247.

17. Kamiza AB, Kamiza S, Mathew CG. HLA-DRB1 alleles and cervical cancer: A meta-analysis of 36 case-control studies. Cancer Epidemiol 2020;67:101748.

18. Wang P, Yang X, Zhou N, et al. Identifying a Potential Key Gene, TIMP1, Associated with Liver Metastases 
of Uveal Melanoma by Weight Gene Co-Expression Network Analysis. Onco Targets Ther 2020;13:11923-34.

19. Wang J, Wen T, Li Z, et al. CD36 upregulates DEK transcription and promotes cell migration and invasion via
GSK-3 $\beta / \beta$-catenin-mediated epithelial-to-mesenchymal transition in gastric cancer. Aging (Albany NY) 2020;13:1883-97.

Cite this article as: Cai GX, Lin L, Zhai XM, Guo ZW, Wu YS, Ye GL, Liu Q, Chen LS, Xing GY, Zhao QH, Tang LL, Mai SH, Ye BJ. A plasma-derived extracellular vesicle mRNA classifier for the detection of breast cancer. Gland Surg 2021;10(6):2002-2009. doi: 10.21037/gs-21-275 
Supplementary

Table S1 Clinical characteristics of the patients and control individuals in this study

\begin{tabular}{|c|c|c|c|}
\hline & Breast cancer pat $(n=144)$ & Benign breast lesion $(n=72)$ & Healthy women $(n=43)$ \\
\hline$<40$ & 27 (18.75\%) & $45(62.5 \%)$ & $25(58.1 \%)$ \\
\hline$>40$ & $117(71.25 \%)$ & $27(37.5 \%)$ & $18(41.9 \%)$ \\
\hline \multicolumn{4}{|l|}{ TNM stage } \\
\hline I & $41(28.47 \%)$ & & \\
\hline II & $70(48.61 \%)$ & & \\
\hline III & $14(9.72 \%)$ & & \\
\hline IV & $3(2.09 \%)$ & & \\
\hline ER/PR- Her2+ & $26(18.06 \%)$ & & \\
\hline ER/PR- Her- & $5(3.47 \%)$ & & \\
\hline
\end{tabular}

Data are $\mathrm{n}, \mathrm{n}(\%)$. Benign breast= patients with benign breast lesions. ER= estrogen receptor. PR= progesterone receptor. Her2= human epidermal growth factor receptor-2. 
Table S2 Primers of the candidate mRNAs

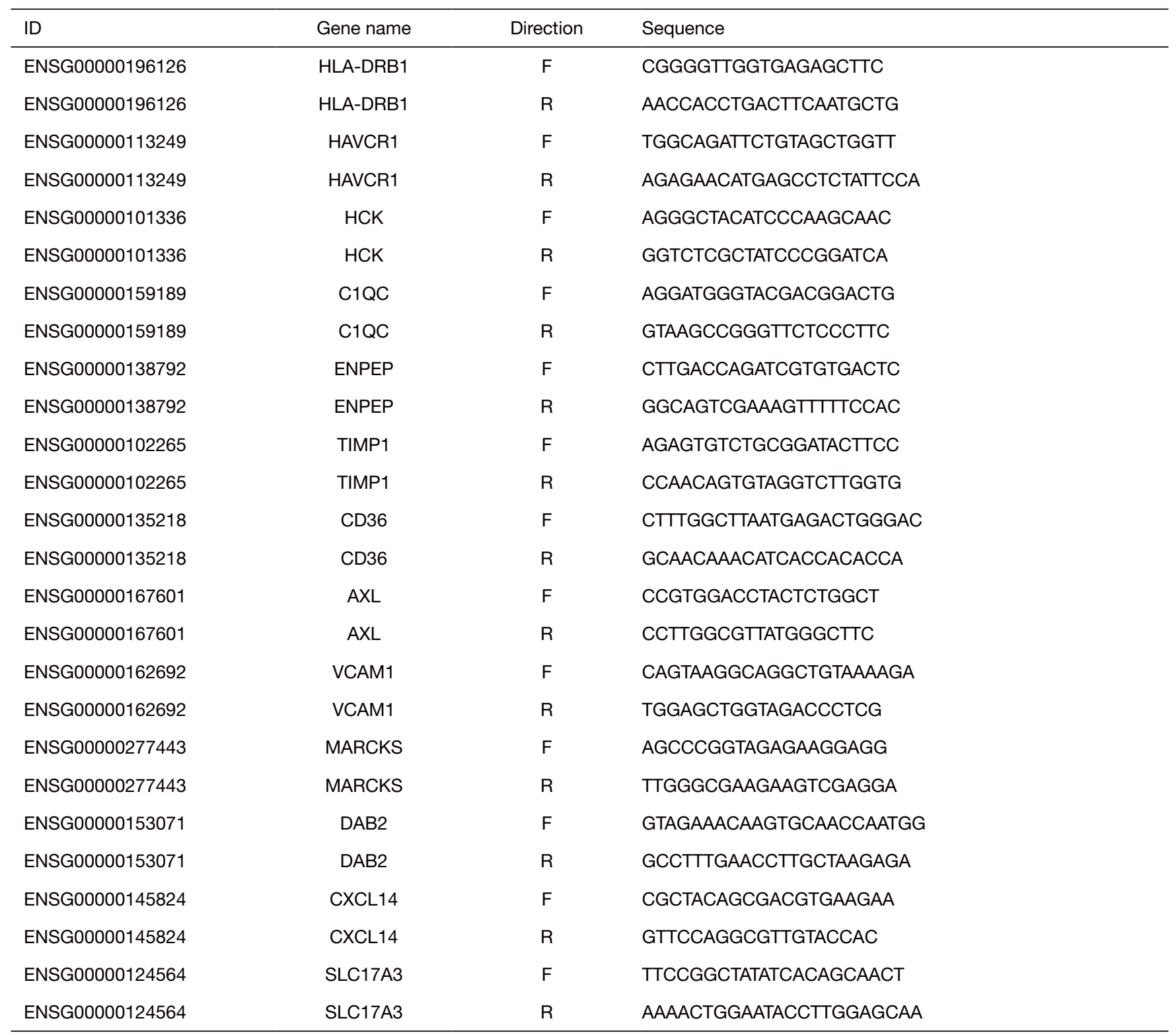

F, forward; R, reverse. 
Table S3 The information of the candidate mRNAs

\begin{tabular}{lcc}
\hline ID & Gene symbol & Threshold \\
\hline ENSG00000196126 & HLA-DRB1 & 0.0761 \\
ENSG00000113249 & HAVCR1 & 0.0087 \\
ENSG00000101336 & HCK & 0.1857 \\
ENSG00000159189 & C1QC & 0.0320 \\
ENSG00000138792 & ENPEP & 0.8527 \\
ENSG00000102265 & TIMP1 & 4.0924 \\
ENSG00000135218 & CD36 & 0.1564 \\
ENSG00000167601 & AXL & 0.5521 \\
ENSG00000162692 & VCAM1 & 0.3120 \\
ENSG00000277443 & MARCKS & 23.635 \\
ENSG00000153071 & DAB2 & 0.3238 \\
ENSG00000145824 & CXCL14 & 1.5475 \\
\hline
\end{tabular}

Threshold, threshold for discretization.

Table S4 mRNAs with significantly different levels

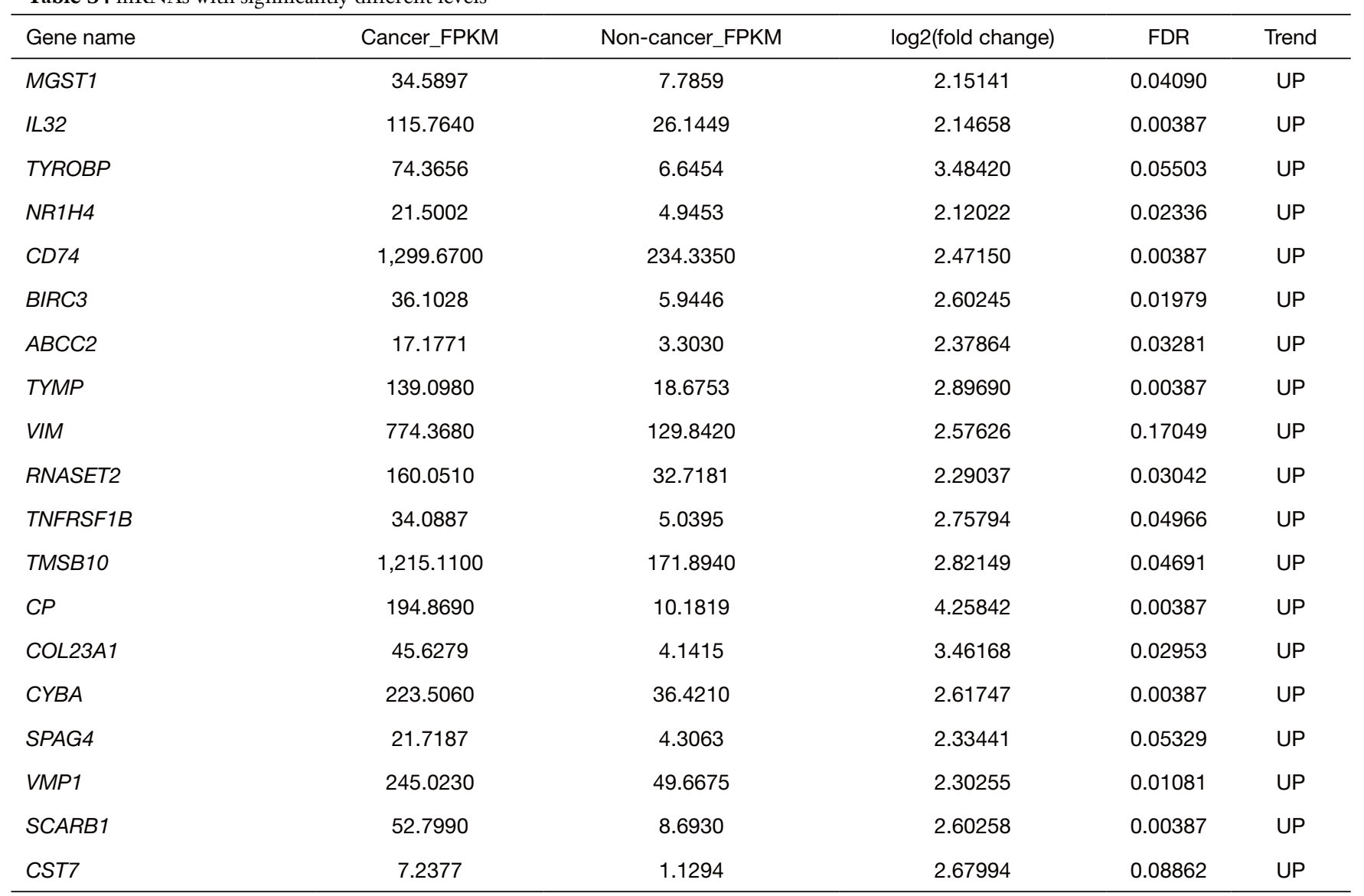

Table S4 (continued) 


\begin{tabular}{|c|c|c|c|c|c|}
\hline Gene name & Cancer_FPKM & Non-cancer_FPKM & $\log 2$ (fold change) & FDR & Trend \\
\hline LRP2 & 121.4210 & 26.8485 & 2.17711 & 0.00387 & UP \\
\hline$S L C 13 A 1$ & 10.0110 & 1.1220 & 3.15748 & 0.00880 & UP \\
\hline PTHLH & 35.8913 & 8.1662 & 2.13590 & 0.13504 & UP \\
\hline RGS1 & 92.5057 & 13.0560 & 2.82483 & 0.00387 & UP \\
\hline$L Y Z$ & 74.2059 & 6.6488 & 3.48038 & 0.15323 & UP \\
\hline ANGPT2 & 16.7873 & 4.1744 & 2.00772 & 0.18836 & UP \\
\hline TREM2 & 12.1972 & 2.2135 & 2.46214 & 0.18519 & UP \\
\hline$S C D$ & 38.7496 & 4.4269 & 3.12981 & 0.00387 & UP \\
\hline HSD3B7 & 33.1037 & 5.7032 & 2.53715 & 0.04691 & UP \\
\hline HMOX1 & 65.8378 & 7.2898 & 3.17497 & 0.00655 & UP \\
\hline APOL1 & 64.0178 & 10.8307 & 2.56335 & 0.00655 & UP \\
\hline TRIB3 & 11.4874 & 2.3127 & 2.31239 & 0.03797 & UP \\
\hline HCK & 13.5973 & 1.5610 & 3.12280 & 0.00387 & UP \\
\hline SAMHD1 & 32.0403 & 6.2232 & 2.36416 & 0.13040 & UP \\
\hline$P G K 1$ & 376.8790 & 81.8157 & 2.20365 & 0.03857 & UP \\
\hline TIMP1 & 195.7460 & 30.0243 & 2.70478 & 0.05077 & UP \\
\hline FLT1 & 90.9207 & 12.1466 & 2.90406 & 0.01399 & UP \\
\hline SLC39A14 & 102.0160 & 18.0885 & 2.49565 & 0.00387 & UP \\
\hline IL27RA & 6.5332 & 1.6080 & 2.02254 & 0.01847 & UP \\
\hline RPS19 & 873.0180 & 210.2250 & 2.05408 & 0.01399 & UP \\
\hline TFEC & 20.7651 & 4.8100 & 2.11004 & 0.00387 & UP \\
\hline SERPINE1 & 50.3593 & 4.6348 & 3.44167 & 0.00387 & UP \\
\hline DDX58 & 24.4537 & 4.3609 & 2.48735 & 0.04691 & UP \\
\hline CUBN & 80.2910 & 16.9641 & 2.24275 & 0.02839 & UP \\
\hline CCND1 & 206.9760 & 41.8340 & 2.30672 & 0.00387 & UP \\
\hline$V W F$ & 122.6810 & 14.2104 & 3.10990 & 0.13066 & UP \\
\hline OAS2 & 48.1845 & 8.3937 & 2.52119 & 0.05784 & UP \\
\hline GAPDH & 950.8090 & 191.5090 & 2.31175 & 0.01081 & UP \\
\hline SOD2 & 404.4800 & 75.9547 & 2.41286 & 0.00387 & UP \\
\hline VEGFA & 630.8220 & 134.3110 & 2.23165 & 0.00387 & UP \\
\hline LOX & 72.3050 & 4.1616 & 4.11887 & 0.00387 & UP \\
\hline SPARC & 770.9060 & 140.2420 & 2.45864 & 0.00387 & UP \\
\hline HAVCR1 & 38.1773 & 2.7356 & 3.80277 & 0.00387 & UP \\
\hline $\mathrm{CDH6}$ & 112.4060 & 18.9697 & 2.56694 & 0.00387 & UP \\
\hline HHLA2 & 22.8002 & 2.3831 & 3.25814 & 0.00387 & UP \\
\hline$F N 1$ & 267.0040 & 65.2055 & 2.03379 & 0.00387 & UP \\
\hline STAT1 & 178.0350 & 41.4442 & 2.10292 & 0.03493 & UP \\
\hline$C D 2$ & 10.8305 & 2.2044 & 2.29662 & 0.04758 & UP \\
\hline SPP1 & 812.8540 & 90.5374 & 3.16641 & 0.00387 & UP \\
\hline NEK6 & 47.4071 & 9.6699 & 2.29353 & 0.00387 & UP \\
\hline IFIT3 & 43.8293 & 8.9774 & 2.28752 & 0.16260 & UP \\
\hline TGFBI & 373.7460 & 43.1515 & 3.11457 & 0.00387 & UP \\
\hline TNFSF 10 & 98.9841 & 21.1184 & 2.22869 & 0.00387 & UP \\
\hline CXCR4 & 50.6256 & 10.3437 & 2.29111 & 0.00387 & UP \\
\hline PRDX4 & 55.1117 & 13.1479 & 2.06752 & 0.07979 & UP \\
\hline$F 13 A 1$ & 36.1219 & 8.6722 & 2.05841 & 0.00655 & UP \\
\hline$S \angle C 17 A 3$ & 101.0000 & 6.4453 & 3.96995 & 0.00387 & UP \\
\hline SLC17A1 & 27.5621 & 4.5971 & 2.58389 & 0.05285 & UP \\
\hline C3 & 378.1060 & 25.2771 & 3.90289 & 0.00387 & UP \\
\hline IFI6 & 603.1450 & 34.4075 & 4.13171 & 0.04654 & UP \\
\hline YEATS4 & 5.6763 & 1.2113 & 2.22844 & 0.17163 & UP \\
\hline$K D R$ & 55.5592 & 13.3704 & 2.05498 & 0.01560 & UP \\
\hline$B B O X 1$ & 43.8473 & 10.6625 & 2.03994 & 0.07588 & UP \\
\hline CD68 & 57.9979 & 8.7557 & 2.72771 & 0.10405 & UP \\
\hline EGLN3 & 155.8510 & 18.4840 & 3.07582 & 0.00387 & UP \\
\hline APOE & 686.9700 & 140.1040 & 2.29375 & 0.00387 & UP \\
\hline ACE2 & 20.3463 & 4.0708 & 2.32137 & 0.06906 & UP \\
\hline PNCK & 100.9930 & 21.3009 & 2.24527 & 0.04379 & UP \\
\hline IDO1 & 14.0941 & 3.4760 & 2.01960 & 0.13096 & UP \\
\hline$A C L Y$ & 80.4375 & 18.0181 & 2.15842 & 0.04758 & UP \\
\hline TOP2A & 5.1601 & 1.2244 & 2.07535 & 0.02109 & UP \\
\hline LRRC41 & 71.1989 & 13.7816 & 2.36911 & 0.00655 & UP \\
\hline TRIM22 & 131.7450 & 27.5914 & 2.25546 & 0.04889 & UP \\
\hline IGJ & 125.3110 & 23.6890 & 2.40322 & 0.16472 & UP \\
\hline XAF1 & 174.3120 & 34.6746 & 2.32973 & 0.00387 & UP \\
\hline EPSTI1 & 43.5537 & 7.2816 & 2.58048 & 0.02439 & UP \\
\hline POSTN & 70.4546 & 7.0390 & 3.32325 & 0.06247 & UP \\
\hline LDHA & 334.3750 & 61.5788 & 2.44096 & 0.01399 & UP \\
\hline CYP2J2 & 22.2407 & 3.5815 & 2.63457 & 0.04691 & UP \\
\hline HAVCR2 & 37.5046 & 5.5127 & 2.76624 & 0.01399 & UP \\
\hline$C D 36$ & 48.2074 & 7.1444 & 2.75436 & 0.00387 & UP \\
\hline UGT2A3 & 28.1931 & 4.1780 & 2.75446 & 0.00387 & UP \\
\hline HILPDA & 96.2785 & 10.8342 & 3.15161 & 0.05609 & UP \\
\hline
\end{tabular}

Table S4 (continued) 


\begin{tabular}{|c|c|c|c|c|c|}
\hline Gene name & Cancer_FPKM & Non-cancer_FPKM & $\log 2$ (fold change) & FDR & Trend \\
\hline AGT & 22.2476 & 4.7454 & 2.22907 & 0.01241 & UP \\
\hline$\angle C P 1$ & 60.6637 & 14.3829 & 2.07648 & 0.00387 & UP \\
\hline MYC & 29.9008 & 7.0586 & 2.08273 & 0.01081 & UP \\
\hline DDX60 & 27.9295 & 5.8466 & 2.25612 & 0.16313 & UP \\
\hline MMP7 & 200.7670 & 16.7297 & 3.58504 & 0.11052 & UP \\
\hline IFI44L & 117.8210 & 15.4851 & 2.92764 & 0.00387 & UP \\
\hline IFI44 & 105.6320 & 15.0976 & 2.80666 & 0.00387 & UP \\
\hline ENPEP & 82.4968 & 8.7917 & 3.23012 & 0.00387 & UP \\
\hline CLEC18B & 29.7152 & 6.4688 & 2.19964 & 0.05503 & UP \\
\hline IFITM3 & 251.9190 & 58.0880 & 2.11665 & 0.07426 & UP \\
\hline SLC2A5 & 52.8797 & 8.1676 & 2.69473 & 0.00387 & UP \\
\hline RGS5 & 601.9250 & 77.4167 & 2.95887 & 0.06298 & UP \\
\hline RIT1 & 25.8044 & 5.7346 & 2.16986 & 0.01712 & UP \\
\hline DEGS1 & 38.0363 & 8.7927 & 2.11300 & 0.10001 & UP \\
\hline NAT8 & 117.6200 & 23.4551 & 2.32615 & 0.00387 & UP \\
\hline IGFBP3 & 514.7070 & 95.0231 & 2.43740 & 0.00387 & UP \\
\hline TMEM27 & 26.8905 & 4.2198 & 2.67184 & 0.16585 & UP \\
\hline PLIN2 & 243.6360 & 15.5610 & 3.96872 & 0.00387 & UP \\
\hline$A D M$ & 112.9750 & 23.0466 & 2.29338 & 0.01712 & UP \\
\hline PDK1 & 31.2311 & 7.3189 & 2.09328 & 0.04889 & UP \\
\hline GJA1 & 34.8822 & 7.6521 & 2.18856 & 0.00387 & UP \\
\hline PLOD2 & 97.0631 & 17.6757 & 2.45715 & 0.00880 & UP \\
\hline ENPP3 & 80.3724 & 1.4419 & 5.80067 & 0.00387 & UP \\
\hline$S L C 16 A 1$ & 26.0676 & 5.6168 & 2.21444 & 0.01847 & UP \\
\hline$S L C 28 A 1$ & 54.6315 & 11.5184 & 2.24579 & 0.04829 & UP \\
\hline ARSE & 20.2463 & 3.9607 & 2.35384 & 0.00387 & UP \\
\hline$M \times 1$ & 144.4400 & 25.5713 & 2.49788 & 0.00387 & UP \\
\hline FCER1G & 32.3932 & 6.3239 & 2.35680 & 0.17569 & UP \\
\hline$C 1 Q C$ & 154.9240 & 20.7497 & 2.90040 & 0.00387 & UP \\
\hline CCDC58 & 13.5701 & 2.9137 & 2.21954 & 0.18766 & UP \\
\hline LYGE & 166.3090 & 30.0716 & 2.46740 & 0.01399 & UP \\
\hline VCAM1 & 156.3420 & 19.3755 & 3.01240 & 0.00387 & UP \\
\hline CTSS & 114.5150 & 13.3406 & 3.10164 & 0.06727 & UP \\
\hline DTX3L & 24.3202 & 5.0667 & 2.26304 & 0.08130 & UP \\
\hline CTSB & 321.0000 & 76.5537 & 2.06803 & 0.00387 & UP \\
\hline$A B C A 1$ & 45.3039 & 10.2325 & 2.14647 & 0.00387 & UP \\
\hline CYBB & 42.1811 & 5.3641 & 2.97519 & 0.09977 & UP \\
\hline CLDN2 & 33.5532 & 8.2297 & 2.02754 & 0.14757 & UP \\
\hline FOLR2 & 17.5791 & 4.1882 & 2.06945 & 0.01560 & UP \\
\hline C2 & 27.0740 & 5.6498 & 2.26065 & 0.00655 & UP \\
\hline$B 2 M$ & $3,895.9300$ & 950.3010 & 2.03551 & 0.10296 & UP \\
\hline NNMT & 356.3260 & 17.6845 & 4.33264 & 0.00387 & UP \\
\hline$A X L$ & 30.4549 & 5.9746 & 2.34976 & 0.00387 & UP \\
\hline SERPINF2 & 76.1275 & 13.5064 & 2.49477 & 0.03336 & UP \\
\hline ANGPTL4 & 152.3840 & 3.0577 & 5.63914 & 0.00387 & UP \\
\hline FTH1 & 771.5220 & 192.6340 & 2.00185 & 0.05193 & UP \\
\hline COL3A1 & 134.2320 & 30.8072 & 2.12339 & 0.03431 & UP \\
\hline SLC16A4 & 32.4636 & 7.2820 & 2.15642 & 0.05609 & UP \\
\hline LGALS9 & 41.0111 & 9.6804 & 2.08288 & 0.01399 & UP \\
\hline CD52 & 24.5403 & 1.8522 & 3.72788 & 0.11660 & UP \\
\hline CD14 & 81.9753 & 16.0484 & 2.35276 & 0.00387 & UP \\
\hline UGT2B7 & 64.1100 & 15.1784 & 2.07853 & 0.00387 & UP \\
\hline$F G G$ & 129.9220 & 4.8828 & 4.73380 & 0.00387 & UP \\
\hline$F G A$ & 87.4698 & 9.4040 & 3.21743 & 0.03135 & UP \\
\hline$F G B$ & 173.7940 & 8.5157 & 4.35111 & 0.00387 & UP \\
\hline SMPDL3A & 21.0100 & 3.7245 & 2.49595 & 0.00387 & UP \\
\hline PARP14 & 87.4088 & 20.3591 & 2.10211 & 0.02109 & UP \\
\hline$C 1 Q B$ & 203.3510 & 19.9941 & 3.34633 & 0.00387 & UP \\
\hline$C 1 Q A$ & 161.5500 & 15.1561 & 3.41402 & 0.00387 & UP \\
\hline KCNE3 & 34.8466 & 8.2107 & 2.08545 & 0.02336 & UP \\
\hline BNIP3 & 78.9625 & 12.9485 & 2.60839 & 0.10042 & UP \\
\hline SAMD9L & 34.4323 & 8.3936 & 2.03640 & 0.00387 & UP \\
\hline C5ort46 & 49.1221 & 4.4939 & 3.45032 & 0.18606 & UP \\
\hline HLA-DQB1 & 66.0857 & 3.7285 & 4.14768 & 0.02336 & UP \\
\hline TMEM45A & 15.0556 & 3.7100 & 2.02081 & 0.12693 & UP \\
\hline HIST1H1B & 39.8999 & 5.8602 & 2.76736 & 0.00387 & UP \\
\hline MAP7D2 & 19.7461 & 1.5418 & 3.67886 & 0.06906 & UP \\
\hline PROS1 & 27.4218 & 5.1654 & 2.40838 & 0.03573 & UP \\
\hline USP18 & 10.8937 & 2.0081 & 2.43957 & 0.00387 & UP \\
\hline NDUFA4L2 & 224.3770 & 31.0639 & 2.85262 & 0.01847 & UP \\
\hline IFITM1 & 184.1950 & 29.0650 & 2.66388 & 0.03281 & UP \\
\hline ISG15 & 202.5720 & 12.7281 & 3.99235 & 0.00387 & UP \\
\hline RAB42 & 13.4298 & 1.8708 & 2.84368 & 0.14176 & UP \\
\hline
\end{tabular}

Table S4 (continued) 
Table S4 (continued)

\begin{tabular}{|c|c|c|c|c|c|}
\hline Gene name & Cancer_FPKM & Non-cancer_FPKM & $\log 2$ (fold change) & FDR & Trend \\
\hline HLA-DRB1 & 160.2480 & 9.2275 & 4.11823 & 0.00387 & UP \\
\hline AKR1C3 & 39.7805 & 9.0105 & 2.14237 & 0.01081 & UP \\
\hline HLA-DQA1 & 65.7906 & 3.6522 & 4.17104 & 0.10644 & UP \\
\hline HIST1H2AI & 24.5722 & 1.3992 & 4.13436 & 0.11101 & UP \\
\hline SERPINA1 & 417.0610 & 46.2690 & 3.17214 & 0.00387 & UP \\
\hline NMB & 11.7954 & 2.3717 & 2.31426 & 0.07136 & UP \\
\hline HIST1H4K & 29.9046 & 3.4400 & 3.11989 & 0.15371 & UP \\
\hline HLA-DRB5 & 80.5012 & 4.9194 & 4.03247 & 0.00387 & UP \\
\hline FCGR3A & 45.7923 & 7.9537 & 2.52540 & 0.01399 & UP \\
\hline HLA-DRA & 501.8820 & 99.8957 & 2.32885 & 0.05503 & UP \\
\hline C4orf47 & 6.9326 & 1.2848 & 2.43190 & 0.09874 & UP \\
\hline CFI & 101.4710 & 17.9410 & 2.49974 & 0.00387 & UP \\
\hline HLA-A & 564.7830 & 135.6480 & 2.05782 & 0.06343 & UP \\
\hline IFI30 & 129.4920 & 18.5807 & 2.80098 & 0.01560 & UP \\
\hline HLA-DPA1 & 279.3960 & 48.7119 & 2.51997 & 0.03135 & UP \\
\hline$H L A-B$ & $1,034.6100$ & 223.9470 & 2.20785 & 0.02109 & UP \\
\hline$E C S C R$ & 24.8675 & 2.8480 & 3.12622 & 0.13220 & UP \\
\hline IGLL5 & 29.8796 & 5.3009 & 2.49484 & 0.06906 & UP \\
\hline CYP26B1 & 3.0910 & 18.7295 & -2.59918 & 0.01241 & DOWN \\
\hline SLC22A16 & 1.3694 & 13.3725 & -3.28765 & 0.00655 & DOWN \\
\hline HOXA11 & 2.0416 & 8.7784 & -2.10427 & 0.19448 & DOWN \\
\hline MPO & 1.3813 & 6.9210 & -2.32496 & 0.09227 & DOWN \\
\hline sox8 & 1.0108 & 6.3433 & -2.64969 & 0.04517 & DOWN \\
\hline YBX2 & 1.7081 & 7.5189 & -2.13813 & 0.06153 & DOWN \\
\hline$A B C C 8$ & 5.6162 & 30.5069 & -2.44148 & 0.00387 & DOWN \\
\hline TAC1 & 1.2663 & 8.2021 & -2.69542 & 0.08011 & DOWN \\
\hline$D L X 6$ & 1.6756 & 11.0378 & -2.71972 & 0.03737 & DOWN \\
\hline DNAH9 & 2.8928 & 12.4486 & -2.10546 & 0.01081 & DOWN \\
\hline SCN4A & 2.3984 & 22.0327 & -3.19950 & 0.06454 & DOWN \\
\hline PAX6 & 4.1243 & 33.3219 & -3.01424 & 0.00387 & DOWN \\
\hline DLEC1 & 1.8712 & 9.4119 & -2.33049 & 0.05960 & DOWN \\
\hline CELSR3 & 1.6305 & 8.1060 & -2.31366 & 0.03042 & DOWN \\
\hline HHATL & 1.1667 & 12.4337 & -3.41373 & 0.03336 & DOWN \\
\hline PRSS3 & 1.0359 & 17.7452 & -4.09848 & 0.00387 & DOWN \\
\hline DPF1 & 1.3298 & 6.8195 & -2.35844 & 0.03857 & DOWN \\
\hline SYT7 & 1.4839 & 9.2377 & -2.63819 & 0.01081 & DOWN \\
\hline STMN4 & 1.6734 & 10.9329 & -2.70782 & 0.01081 & DOWN \\
\hline SRCIN1 & 1.7379 & 8.0918 & -2.21914 & 0.05960 & DOWN \\
\hline ATP1A2 & 4.7962 & 27.1310 & -2.49997 & 0.01241 & DOWN \\
\hline PLEKHB1 & 5.9779 & 33.4537 & -2.48444 & 0.00387 & DOWN \\
\hline CPS1 & 3.4790 & 25.2046 & -2.85694 & 0.02225 & DOWN \\
\hline KCNG1 & 1.8623 & 8.4383 & -2.17988 & 0.05193 & DOWN \\
\hline CYP46A1 & 2.1301 & 9.0161 & -2.08162 & 0.00880 & DOWN \\
\hline RTN4R & 1.6590 & 9.7312 & -2.55234 & 0.02225 & DOWN \\
\hline MYO16 & 1.6216 & 12.5611 & -2.95352 & 0.04758 & DOWN \\
\hline$Z P B P$ & 2.1409 & 13.8069 & -2.68913 & 0.10405 & DOWN \\
\hline$T G$ & 2.8836 & 23.0669 & -2.99988 & 0.00387 & DOWN \\
\hline LMO3 & 4.4808 & 26.2613 & -2.55110 & 0.00387 & DOWN \\
\hline CBLN4 & 1.3598 & 6.7726 & -2.31630 & 0.01081 & DOWN \\
\hline KCNH2 & 1.3715 & 8.1734 & -2.57522 & 0.04013 & DOWN \\
\hline$|T| H 1$ & 1.6599 & 10.5544 & -2.66870 & 0.06678 & DOWN \\
\hline CAMК2B & 1.9588 & 11.6774 & -2.57568 & 0.00387 & DOWN \\
\hline$P S D$ & 2.3896 & 15.0884 & -2.65859 & 0.02109 & DOWN \\
\hline RIMBP2 & 3.4553 & 16.2699 & -2.23532 & 0.03857 & DOWN \\
\hline DGAT2 & 2.0967 & 10.8469 & -2.37109 & 0.02109 & DOWN \\
\hline SEZ6 & 2.3938 & 26.9105 & -3.49079 & 0.03281 & DOWN \\
\hline SLC6A16 & 1.4369 & 6.4578 & -2.16807 & 0.02839 & DOWN \\
\hline AHRR & 2.2393 & 10.1016 & -2.17345 & 0.03655 & DOWN \\
\hline ATP2C2 & 2.6490 & 23.7868 & -3.16663 & 0.00387 & DOWN \\
\hline SNCAIP & 3.1894 & 18.9072 & -2.56760 & 0.02109 & DOWN \\
\hline BCAS1 & 2.0446 & 18.0461 & -3.14180 & 0.00387 & DOWN \\
\hline NTN1 & 1.1803 & 6.2401 & -2.40248 & 0.01979 & DOWN \\
\hline ROPN1 & 1.6090 & 14.1842 & -3.14001 & 0.01241 & DOWN \\
\hline SNAP91 & 1.1722 & 6.7117 & -2.51750 & 0.00880 & DOWN \\
\hline STAG3 & 3.2438 & 18.7223 & -2.52898 & 0.00387 & DOWN \\
\hline SYT1 & 1.6259 & 7.3649 & -2.17942 & 0.01241 & DOWN \\
\hline REEP1 & 2.3510 & 9.7789 & -2.05640 & 0.08263 & DOWN \\
\hline PITX1 & 2.1058 & 11.4884 & -2.44777 & 0.10296 & DOWN \\
\hline SPTB & 2.7678 & 14.2011 & -2.35917 & 0.04187 & DOWN \\
\hline FGF10 & 1.2978 & 10.5327 & -3.02080 & 0.16675 & DOWN \\
\hline FRMPD1 & 1.1134 & 5.5981 & -2.32995 & 0.12207 & DOWN \\
\hline TRIP13 & 1.2535 & 7.4873 & -2.57845 & 0.15824 & DOWN \\
\hline МҮОЗВ & 2.0613 & 9.1637 & -2.15236 & 0.09102 & DOWN \\
\hline
\end{tabular}

Table S4 (continued) 
Table S4 (continued)

\begin{tabular}{|c|c|c|c|c|c|}
\hline Gene name & Cancer_FPKM & Non-cancer_FPKM & $\log 2$ (fold change) & FDR & Trend \\
\hline ASIC4 & 1.1837 & 9.8864 & -3.06211 & 0.00387 & DOWN \\
\hline SPEG & 6.2169 & 32.2467 & -2.37488 & 0.00387 & DOWN \\
\hline MOV10L1 & 1.5093 & 6.6611 & -2.14185 & 0.05767 & DOWN \\
\hline PPP2R2C & 1.8533 & 7.8806 & -2.08823 & 0.02839 & DOWN \\
\hline$S L C 12 A 1$ & 109.5020 & 529.6760 & -2.27416 & 0.00387 & DOWN \\
\hline WSCD2 & 3.5772 & 15.9689 & -2.15835 & 0.00387 & DOWN \\
\hline$A D D 2$ & 1.9214 & 11.2586 & -2.55083 & 0.00387 & DOWN \\
\hline CACNG5 & 1.7344 & 12.3678 & -2.83405 & 0.16106 & DOWN \\
\hline$A M P H$ & 2.1427 & 10.3787 & -2.27614 & 0.01560 & DOWN \\
\hline RBFOX1 & 1.3333 & 8.2257 & -2.62512 & 0.00387 & DOWN \\
\hline MYH7B & 2.8458 & 13.5848 & -2.25507 & 0.06642 & DOWN \\
\hline TP73 & 2.1191 & 11.3227 & -2.41767 & 0.03655 & DOWN \\
\hline KCNN2 & 1.4442 & 9.5661 & -2.72762 & 0.00387 & DOWN \\
\hline TCF7 & 7.1703 & 31.8537 & -2.15135 & 0.03493 & DOWN \\
\hline PKP1 & 2.3617 & 11.2197 & -2.24816 & 0.03281 & DOWN \\
\hline$P G R$ & 1.7933 & 8.1755 & -2.18868 & 0.00387 & DOWN \\
\hline SERTAD4 & 1.4107 & 7.1556 & -2.34263 & 0.01241 & DOWN \\
\hline GRHL2 & 3.1650 & 15.9456 & -2.33289 & 0.00880 & DOWN \\
\hline HAL & 1.9254 & 16.5661 & -3.10499 & 0.01241 & DOWN \\
\hline SLCO1A2 & 1.8682 & 9.5488 & -2.35369 & 0.00387 & DOWN \\
\hline GCKR & 1.4177 & 8.5367 & -2.59013 & 0.13203 & DOWN \\
\hline IGSF9 & 1.1180 & 11.3487 & -3.34352 & 0.02225 & DOWN \\
\hline HSD17B2 & 2.6034 & 14.9055 & -2.51737 & 0.06454 & DOWN \\
\hline PHACTR3 & 1.5533 & 7.0366 & -2.17957 & 0.05767 & DOWN \\
\hline SULT2B1 & 1.2391 & 6.8556 & -2.46800 & 0.05710 & DOWN \\
\hline SLC4A11 & 2.3926 & 17.5294 & -2.87313 & 0.03336 & DOWN \\
\hline$F 11$ & 4.8720 & 23.2757 & -2.25624 & 0.09481 & DOWN \\
\hline C20orf26 & 1.5857 & 8.6946 & -2.45501 & 0.01847 & DOWN \\
\hline RPH $3 A$ & 1.4390 & 9.1772 & -2.67299 & 0.00387 & DOWN \\
\hline CHGB & 1.6753 & 10.4980 & -2.64760 & 0.13787 & DOWN \\
\hline NOS1 & 2.8431 & 12.9713 & -2.18978 & 0.07087 & DOWN \\
\hline OTUB2 & 1.0897 & 8.0401 & -2.88332 & 0.00655 & DOWN \\
\hline LAMB4 & 1.0852 & 5.9786 & -2.46188 & 0.04437 & DOWN \\
\hline MYO15A & 2.2216 & 14.9373 & -2.74924 & 0.00387 & DOWN \\
\hline PITPNM3 & 1.7085 & 8.8208 & -2.36821 & 0.00655 & DOWN \\
\hline ZFHX4 & 2.6046 & 14.7363 & -2.50025 & 0.00387 & DOWN \\
\hline CPA1 & 1.0046 & 4.2923 & -2.09512 & 0.03336 & DOWN \\
\hline TGM1 & 4.6901 & 29.7509 & -2.66525 & 0.03493 & DOWN \\
\hline TBX15 & 1.0859 & 6.3469 & -2.54711 & 0.02537 & DOWN \\
\hline GABRP & 1.3165 & 6.5651 & -2.31813 & 0.01847 & DOWN \\
\hline NXPE1 & 1.0773 & 6.0652 & -2.49319 & 0.06153 & DOWN \\
\hline EPB41L4B & 1.0017 & 4.0771 & -2.02513 & 0.15744 & DOWN \\
\hline P2RX6 & 1.2233 & 8.8644 & -2.85727 & 0.01847 & DOWN \\
\hline SEPT3 & 1.0069 & 5.5181 & -2.45421 & 0.01560 & DOWN \\
\hline PVALB & 2.1664 & 8.9013 & -2.03873 & 0.17987 & DOWN \\
\hline KCTD17 & 7.3519 & 29.9049 & -2.02419 & 0.00387 & DOWN \\
\hline KCNK10 & 1.1417 & 7.7489 & -2.76283 & 0.01399 & DOWN \\
\hline $\mathrm{COCH}$ & 2.3752 & 17.8996 & -2.91383 & 0.13596 & DOWN \\
\hline C14orf166B & 1.4759 & 14.9637 & -3.34184 & 0.16675 & DOWN \\
\hline CHGA & 3.1319 & 25.0115 & -2.99748 & 0.03655 & DOWN \\
\hline ASB2 & 3.1718 & 17.1568 & -2.43540 & 0.01081 & DOWN \\
\hline SLC8A3 & 1.8012 & 17.9564 & -3.31744 & 0.00387 & DOWN \\
\hline TCL1A & 1.6218 & 8.0747 & -2.31584 & 0.05329 & DOWN \\
\hline DHRS2 & 1.8375 & 12.3589 & -2.74971 & 0.00387 & DOWN \\
\hline CPNE6 & 3.5985 & 16.8268 & -2.22530 & 0.02537 & DOWN \\
\hline$v S \times 1$ & 2.7529 & 14.5057 & -2.39758 & 0.06298 & DOWN \\
\hline SALL4 & 1.1544 & 5.1732 & -2.16396 & 0.02336 & DOWN \\
\hline BMP7 & 2.6634 & 13.2424 & -2.31380 & 0.03573 & DOWN \\
\hline COL2OA1 & 1.5632 & 18.1895 & -3.54053 & 0.00387 & DOWN \\
\hline SNPH & 2.3343 & 10.2750 & -2.13805 & 0.01560 & DOWN \\
\hline$B P I$ & 1.1603 & 4.8070 & -2.05064 & 0.13242 & DOWN \\
\hline EPPIN & 1.0667 & 16.4687 & -3.94846 & 0.00387 & DOWN \\
\hline CELF4 & 1.6587 & 8.7628 & -2.40136 & 0.00387 & DOWN \\
\hline STBSIA5 & 2.5903 & 12.3899 & -2.25800 & 0.01847 & DOWN \\
\hline NOL4 & 1.9652 & 12.7326 & -2.69581 & 0.01560 & DOWN \\
\hline VGLL1 & 1.1705 & 13.9529 & -3.57538 & 0.05024 & DOWN \\
\hline CBLN1 & 1.2237 & 10.7122 & -3.12996 & 0.07803 & DOWN \\
\hline$C C D C 113$ & 3.3417 & 13.9269 & -2.05920 & 0.03655 & DOWN \\
\hline NDRG4 & 5.9939 & 36.3870 & -2.60186 & 0.00387 & DOWN \\
\hline HAS3 & 2.0123 & 12.3935 & -2.62268 & 0.09506 & DOWN \\
\hline SMPD3 & 4.3455 & 25.1374 & -2.53224 & 0.00387 & DOWN \\
\hline ACSBG1 & 2.8344 & 19.8809 & -2.81025 & 0.00655 & DOWN \\
\hline IGDCC4 & 1.8913 & 13.1482 & -2.79744 & 0.04517 & DOWN \\
\hline
\end{tabular}

Table S4 (continued) 


\begin{tabular}{|c|c|c|c|c|c|}
\hline Gene name & Cancer_FPKM & Non-cancer_FPKM & $\log 2$ (fold change) & FDR & Trend \\
\hline FAM189A1 & 1.7313 & 9.5470 & -2.46322 & 0.07588 & DOWN \\
\hline TRPA1 & 2.5965 & 12.9199 & -2.31498 & 0.16726 & DOWN \\
\hline SLC17A7 & 1.1830 & 5.7142 & -2.27208 & 0.08535 & DOWN \\
\hline SLC1AG & 1.3937 & 7.1954 & -2.36814 & 0.00880 & DOWN \\
\hline$C R X$ & 2.3051 & 14.7386 & -2.67668 & 0.02109 & DOWN \\
\hline$M A G$ & 1.1945 & 5.0030 & -2.06634 & 0.17581 & DOWN \\
\hline PON3 & 1.3881 & 9.8221 & -2.82292 & 0.01560 & DOWN \\
\hline RNF32 & 2.2291 & 9.3946 & -2.07536 & 0.00655 & DOWN \\
\hline VIPR2 & 1.1126 & 6.4818 & -2.54251 & 0.08828 & DOWN \\
\hline$E V X 1$ & 1.6654 & 16.6538 & -3.32192 & 0.05024 & DOWN \\
\hline GCK & 1.0033 & 6.6846 & -2.73605 & 0.02439 & DOWN \\
\hline LHX6 & 1.8979 & 10.8063 & -2.50943 & 0.00387 & DOWN \\
\hline TNFSF8 & 3.8578 & 15.6345 & -2.01890 & 0.03042 & DOWN \\
\hline RLN2 & 1.0839 & 5.3215 & -2.29567 & 0.14980 & DOWN \\
\hline TYRP1 & 3.3192 & 14.6539 & -2.14239 & 0.07175 & DOWN \\
\hline $\mathrm{CDH} 23$ & 3.4339 & 14.2238 & -2.05038 & 0.00655 & DOWN \\
\hline$T L X 1$ & 2.7712 & 19.7351 & -2.83220 & 0.04585 & DOWN \\
\hline KAZALD1 & 2.4044 & 13.1076 & -2.44663 & 0.03493 & DOWN \\
\hline NEURL1 & 1.1953 & 8.5713 & -2.84213 & 0.05548 & DOWN \\
\hline DKK1 & 2.7194 & 17.5987 & -2.69409 & 0.08324 & DOWN \\
\hline SORCS1 & 1.6492 & 11.3797 & -2.78665 & 0.01560 & DOWN \\
\hline KRT23 & 1.9558 & 17.4160 & -3.15460 & 0.05329 & DOWN \\
\hline RUNDC3A & 1.5347 & 6.3144 & -2.04067 & 0.08571 & DOWN \\
\hline ALDH3A1 & 1.7514 & 8.2470 & -2.23539 & 0.00880 & DOWN \\
\hline ASIC2 & 1.9295 & 9.2204 & -2.25657 & 0.07543 & DOWN \\
\hline HSD17B1 & 1.2412 & 9.1455 & -2.88131 & 0.03431 & DOWN \\
\hline$D L X 4$ & 1.9551 & 21.0465 & -3.42826 & 0.01560 & DOWN \\
\hline$P P Y$ & 1.5227 & 19.2818 & -3.66252 & 0.10109 & DOWN \\
\hline MPP2 & 2.2560 & 12.8531 & -2.51031 & 0.00387 & DOWN \\
\hline VTN & 1.4886 & 9.7736 & -2.71492 & 0.05193 & DOWN \\
\hline CWH43 & 1.6644 & 9.9599 & -2.58115 & 0.17937 & DOWN \\
\hline$C P Z$ & 2.5058 & 10.2586 & -2.03347 & 0.07662 & DOWN \\
\hline GLRB & 1.1574 & 6.0959 & -2.39696 & 0.07175 & DOWN \\
\hline HGFAC & 1.2056 & 6.1539 & -2.35172 & 0.11269 & DOWN \\
\hline DDX25 & 1.8371 & 7.7307 & -2.07317 & 0.14461 & DOWN \\
\hline TECTA & 1.5405 & 7.6739 & -2.31659 & 0.03281 & DOWN \\
\hline B3GAT1 & 3.2185 & 17.8649 & -2.47267 & 0.01712 & DOWN \\
\hline CCKBR & 1.5801 & 14.2872 & -3.17662 & 0.04889 & DOWN \\
\hline$H P X$ & 2.9717 & 19.2018 & -2.69189 & 0.03737 & DOWN \\
\hline PVRL1 & 3.9675 & 21.3114 & -2.42533 & 0.00387 & DOWN \\
\hline C11orf21 & 3.3580 & 13.7685 & -2.03568 & 0.08742 & DOWN \\
\hline PTPN5 & 2.2642 & 10.9707 & -2.27660 & 0.04654 & DOWN \\
\hline$A S I C 1$ & 1.5180 & 6.3586 & -2.06653 & 0.02439 & DOWN \\
\hline TSPAN11 & 1.5624 & 10.4252 & -2.73824 & 0.00387 & DOWN \\
\hline CYP27B1 & 5.4071 & 43.3834 & -3.00421 & 0.02756 & DOWN \\
\hline GLI1 & 1.6937 & 8.2106 & -2.27733 & 0.11729 & DOWN \\
\hline AKAP3 & 2.3294 & 15.0954 & -2.69610 & 0.04305 & DOWN \\
\hline GPR133 & 3.9680 & 17.3906 & -2.13182 & 0.02439 & DOWN \\
\hline GNB3 & 3.1877 & 20.6205 & -2.69351 & 0.02225 & DOWN \\
\hline CDCA3 & 2.5949 & 11.3840 & -2.13326 & 0.02439 & DOWN \\
\hline STRSIA1 & 7.4267 & 32.0619 & -2.11007 & 0.02225 & DOWN \\
\hline$A I C D A$ & 1.1071 & 7.3120 & -2.72345 & 0.12317 & DOWN \\
\hline$R S P H 4 A$ & 1.3133 & 5.5700 & -2.08443 & 0.10001 & DOWN \\
\hline OPRM1 1 & 1.3901 & 13.0415 & -3.22983 & 0.00387 & DOWN \\
\hline TULP1 & 1.8726 & 11.6949 & -2.64281 & 0.05784 & DOWN \\
\hline SLC26A8 & 1.0385 & 5.7549 & -2.47026 & 0.06393 & DOWN \\
\hline MDGA1 & 5.4986 & 22.2251 & -2.01505 & 0.00387 & DOWN \\
\hline RBM24 & 1.4389 & 7.9709 & -2.46980 & 0.06503 & DOWN \\
\hline HDGFL1 & 3.8513 & 19.8409 & -2.36506 & 0.06873 & DOWN \\
\hline EYA4 & 2.4780 & 13.4675 & -2.44223 & 0.01399 & DOWN \\
\hline PRPH2 & 1.2076 & 5.0632 & -2.06796 & 0.01081 & DOWN \\
\hline PRSS16 & 5.0080 & 22.3533 & -2.15817 & 0.00655 & DOWN \\
\hline TBX18 & 1.6055 & 9.9133 & -2.62637 & 0.01081 & DOWN \\
\hline PCDHB7 & 2.1932 & 10.1851 & -2.21535 & 0.03431 & DOWN \\
\hline GRM6 & 2.6070 & 13.0461 & -2.32315 & 0.11234 & DOWN \\
\hline HRG & 2.1675 & 11.5697 & -2.41622 & 0.08904 & DOWN \\
\hline FGF12 & 2.5953 & 19.6215 & -2.91846 & 0.01241 & DOWN \\
\hline ROPN1B & 1.4841 & 16.2465 & -3.45246 & 0.00387 & DOWN \\
\hline KIAA1257 & 1.5640 & 6.3923 & -2.03109 & 0.09524 & DOWN \\
\hline PEX5L & 2.4132 & 13.0085 & -2.43047 & 0.04305 & DOWN \\
\hline ARHGEF26 & 1.7920 & 7.3565 & -2.03746 & 0.10644 & DOWN \\
\hline VIPR1 & 3.2698 & 21.1596 & -2.69406 & 0.00387 & DOWN \\
\hline$S \angle C 3 O A 3$ & 1.8057 & 7.3100 & -2.01733 & 0.06393 & DOWN \\
\hline
\end{tabular}




\begin{tabular}{|c|c|c|c|c|c|}
\hline Gene name & Cancer_FPKM & Non-cancer_FPKM & $\log 2$ (fold change) & FDR & Trend \\
\hline$T \angle X 2$ & 3.0187 & 17.7044 & -2.55210 & 0.15362 & DOWN \\
\hline TACR1 & 1.1881 & 11.0960 & -3.22327 & 0.00655 & DOWN \\
\hline KCNJ13 & 1.4961 & 7.1352 & -2.25374 & 0.17633 & DOWN \\
\hline OTX1 & 1.8918 & 22.6628 & -3.58250 & 0.00655 & DOWN \\
\hline SLC9A2 & 1.4050 & 7.3091 & -2.37914 & 0.01081 & DOWN \\
\hline MLPH & 3.7539 & 18.5709 & -2.30659 & 0.00387 & DOWN \\
\hline KIAA1324 & 3.5714 & 20.5783 & -2.52658 & 0.00655 & DOWN \\
\hline MYCL & 3.2674 & 13.8523 & -2.08391 & 0.07175 & Down \\
\hline ST6GALNAC5 & 1.3174 & 13.4860 & -3.35575 & 0.05233 & DOWN \\
\hline MFAP2 & 2.0810 & 12.9023 & -2.63227 & 0.02225 & DOWN \\
\hline PLA2G2D & 1.3414 & 6.0283 & -2.16800 & 0.04013 & DOWN \\
\hline ARTN & 1.7037 & 11.9966 & -2.81591 & 0.00880 & DOWN \\
\hline PTCH2 & 1.4983 & 7.1732 & -2.25928 & 0.08442 & DOWN \\
\hline PROX1 & 3.0944 & 26.2106 & -3.08242 & 0.01081 & DOWN \\
\hline CENPF & 3.0876 & 13.6576 & -2.14514 & 0.06819 & DOWN \\
\hline MUC5B & 1.6494 & 17.3875 & -3.39801 & 0.02109 & DOWN \\
\hline CA14 & 1.1448 & 7.1664 & -2.64618 & 0.06393 & DOWN \\
\hline ATP10B & 2.6125 & 24.5740 & -3.23365 & 0.00387 & Down \\
\hline CNR1 & 2.1611 & 13.0003 & -2.58870 & 0.03493 & DOWN \\
\hline TRPM6 & 1.0751 & 6.6481 & -2.62850 & 0.00880 & DOWN \\
\hline PPP4R4 & 1.3387 & 6.3430 & -2.24429 & 0.11234 & DOWN \\
\hline ESRRB & 2.6412 & 16.5489 & -2.64748 & 0.01560 & DOWN \\
\hline CRHR1 & 1.2838 & 12.0311 & -3.22824 & 0.01241 & DOWN \\
\hline HOXB1 & 1.1317 & 7.3255 & -2.69442 & 0.16140 & DOWN \\
\hline GRIA2 & 1.5235 & 9.5850 & -2.65335 & 0.02537 & DOWN \\
\hline TP53AIP1 & 1.2891 & 9.2807 & -2.84783 & 0.04187 & DOWN \\
\hline CHRNA2 & 1.0421 & 12.4937 & -3.58370 & 0.00387 & DOWN \\
\hline ADRA1A & 3.6376 & 20.3181 & -2.48170 & 0.00880 & DowN \\
\hline TNFRSF8 & 1.2974 & 5.5470 & -2.09608 & 0.09506 & DOWN \\
\hline TSHZ3 & 5.6430 & 26.2028 & -2.21518 & 0.00880 & DOWN \\
\hline LHX4 & 1.7595 & 7.8812 & -2.16326 & 0.15857 & DOWN \\
\hline GJAB & 2.3815 & 13.7966 & -2.53437 & 0.02537 & DOWN \\
\hline CCRL2 & 4.1546 & 17.6842 & -2.08966 & 0.02109 & DOWN \\
\hline CSMD2 & 1.5428 & 14.2744 & -3.20979 & 0.00387 & DOWN \\
\hline HPCA & 1.6793 & 6.7798 & -2.01339 & 0.15857 & DOWN \\
\hline TBX22 & 1.2173 & 6.5946 & -2.43761 & 0.19961 & DOWN \\
\hline LDB3 & 1.5266 & 8.5554 & -2.48649 & 0.02336 & DOWN \\
\hline KIAA1045 & 1.6337 & 6.6800 & -2.03167 & 0.04141 & DOWN \\
\hline SFTPA1 & 1.5541 & 15.5298 & -3.32086 & 0.01712 & DOWN \\
\hline PLP1 & 2.8596 & 21.2227 & -2.89173 & 0.00387 & DOWN \\
\hline FAM124B & 1.6522 & 9.9380 & -2.58856 & 0.05233 & DOWN \\
\hline CTCFL & 1.0134 & 6.6880 & -2.72240 & 0.00387 & DOWN \\
\hline WFDC3 & 1.2385 & 9.7831 & -2.98169 & 0.00387 & Down \\
\hline KCNS1 & 1.1899 & 9.7758 & -3.03837 & 0.02756 & DOWN \\
\hline SLC12A5 & 1.7113 & 7.6786 & -2.16573 & 0.06678 & DOWN \\
\hline GDAP1L1 & 1.3788 & 7.1787 & -2.38035 & 0.09328 & DOWN \\
\hline CHST8 & 1.4334 & 6.9560 & -2.27888 & 0.17068 & DOWN \\
\hline PSG8 & 1.1794 & 12.2851 & -3.38081 & 0.00387 & DOWN \\
\hline GRM4 & 1.7398 & 16.7494 & -3.26716 & 0.00387 & DOWN \\
\hline PACSIN1 & 1.9015 & 15.2759 & -3.00609 & 0.01081 & DOWN \\
\hline KLHL31 & 1.6047 & 6.6500 & -2.05101 & 0.14461 & DOWN \\
\hline C1orf61 & 3.3538 & 25.5598 & -2.93001 & 0.00387 & DOWN \\
\hline PAX1 & 1.5205 & 9.1687 & -2.59213 & 0.06954 & DOWN \\
\hline BFSP1 & 1.0077 & 6.1079 & -2.59967 & 0.07244 & DOWN \\
\hline$F 10$ & 2.6213 & 11.0462 & -2.07518 & 0.15149 & DOWN \\
\hline$S I X 1$ & 1.1772 & 9.7699 & -3.05303 & 0.02648 & DOWN \\
\hline AVPR2 & 2.2449 & 10.5280 & -2.22952 & 0.16933 & DOWN \\
\hline BEST3 & 1.1173 & 12.1637 & -3.44456 & 0.00387 & DOWN \\
\hline SYNGR3 & 1.2633 & 8.8668 & -2.81119 & 0.06557 & DOWN \\
\hline PKMYT1 & 4.9335 & 22.8218 & -2.20974 & 0.05077 & DOWN \\
\hline RASL11B & 2.9672 & 12.6358 & -2.09037 & 0.14501 & DOWN \\
\hline GNAZ & 2.7866 & 12.5731 & -2.17375 & 0.03573 & DOWN \\
\hline C22ort23 & 1.0953 & 5.0876 & -2.21567 & 0.08504 & DowN \\
\hline KRT17 & 2.7583 & 25.8195 & -3.22659 & 0.07288 & DOWN \\
\hline KCNC1 & 1.3781 & 14.8700 & -3.43169 & 0.00387 & DOWN \\
\hline AIPL1 & 1.0249 & 4.9249 & -2.26465 & 0.09505 & DOWN \\
\hline FOXA1 & 2.4016 & 18.1722 & -2.91964 & 0.05285 & DOWN \\
\hline$C D O 1$ & 3.0122 & 21.8974 & -2.86186 & 0.00387 & DOWN \\
\hline CHRNA10 & 2.2441 & 9.1800 & -2.03236 & 0.19597 & DOWN \\
\hline SYT5 & 2.0525 & 9.1108 & -2.15022 & 0.05819 & DOWN \\
\hline TNNI3 & 1.1990 & 7.9130 & -2.72243 & 0.00655 & DOWN \\
\hline PRRG3 & 1.4845 & 10.0943 & -2.76545 & 0.00655 & DOWN \\
\hline KCNA5 & 1.7083 & 8.1784 & -2.25923 & 0.01241 & DOWN \\
\hline
\end{tabular}




\begin{tabular}{|c|c|c|c|c|c|}
\hline Gene name & Cancer_FPKM & Non-cancer_FPKM & $\log 2$ (fold change) & FDR & Trend \\
\hline DPP6 & 4.6969 & 25.6340 & -2.44829 & 0.00387 & DOWN \\
\hline KIF1A & 4.0325 & 33.4428 & -3.05196 & 0.00387 & DOWN \\
\hline OLFM1 & 4.7566 & 21.0473 & -2.14564 & 0.01712 & DOWN \\
\hline TNNT3 & 1.5253 & 7.3747 & -2.27352 & 0.16066 & DOWN \\
\hline CYP2E1 & 3.5714 & 18.0379 & -2.33646 & 0.03493 & DOWN \\
\hline$M N X 1$ & 2.8898 & 15.2360 & -2.39844 & 0.01712 & DOWN \\
\hline DUSP9 & 10.8989 & 44.8771 & -2.04180 & 0.02225 & DOWN \\
\hline AOC2 & 1.2349 & 5.7629 & -2.22236 & 0.04829 & DOWN \\
\hline TNS4 & 1.1484 & 6.3839 & -2.47480 & 0.09527 & DOWN \\
\hline$A B H D 12 B$ & 1.4253 & 8.5695 & -2.58797 & 0.03737 & DOWN \\
\hline C19orf57 & 2.4076 & 9.8930 & -2.03884 & 0.06393 & DOWN \\
\hline SLC6A11 & 1.0407 & 9.3343 & -3.16497 & 0.04141 & DOWN \\
\hline$B C A N$ & 1.4117 & 12.2677 & -3.11934 & 0.02109 & DOWN \\
\hline CRP & 1.2323 & 9.1689 & -2.89544 & 0.01847 & DOWN \\
\hline HAPLN2 & 1.3365 & 5.7609 & -2.10781 & 0.18026 & DOWN \\
\hline CHIT1 & 4.1886 & 21.1536 & -2.33638 & 0.02109 & DOWN \\
\hline LGR6 & 1.4803 & 15.4746 & -3.38598 & 0.00387 & DOWN \\
\hline TMCC2 & 1.8130 & 7.2575 & -2.00108 & 0.02336 & DOWN \\
\hline UNC79 & 1.0639 & 4.9754 & -2.22542 & 0.09661 & DOWN \\
\hline GSTM5 & 1.9951 & 15.6517 & -2.97180 & 0.01399 & DOWN \\
\hline SYT6 & 2.5998 & 11.2324 & -2.11119 & 0.02439 & DOWN \\
\hline$C D 101$ & 2.0976 & 13.4868 & -2.68471 & 0.05784 & DOWN \\
\hline CRB1 & 1.4409 & 8.2433 & -2.51630 & 0.01081 & DOWN \\
\hline$R A X$ & 2.9534 & 22.9234 & -2.95636 & 0.12028 & DOWN \\
\hline MTNR1B & 1.1313 & 11.9263 & -3.39807 & 0.07203 & DOWN \\
\hline$S P O C D 1$ & 2.4917 & 12.3777 & -2.31254 & 0.02336 & DOWN \\
\hline ELF5 & 1.8151 & 18.6713 & -3.36273 & 0.05548 & DOWN \\
\hline$P R P H$ & 1.5892 & 7.5234 & -2.24314 & 0.08862 & DOWN \\
\hline AMHR2 & 1.1524 & 7.1074 & -2.62464 & 0.02839 & DOWN \\
\hline FAM186B & 1.4815 & 9.1388 & -2.62494 & 0.08862 & DOWN \\
\hline FAIM2 & 1.5631 & 9.1221 & -2.54493 & 0.00387 & DOWN \\
\hline $\mathrm{KCNH3}$ & 1.1557 & 5.6944 & -2.30076 & 0.10529 & DOWN \\
\hline EGR4 & 1.0223 & 6.9752 & -2.77037 & 0.07979 & DOWN \\
\hline SLC9A5 & 1.2194 & 5.6401 & -2.20955 & 0.04691 & DOWN \\
\hline GPR55 & 1.2118 & 9.1052 & -2.90952 & 0.00387 & DOWN \\
\hline PAX3 & 1.6292 & 11.8382 & -2.86120 & 0.01241 & DOWN \\
\hline WNT10A & 2.1429 & 8.7279 & -2.02604 & 0.01241 & DOWN \\
\hline ARHGEF4 & 2.5924 & 23.6024 & -3.18659 & 0.00387 & DOWN \\
\hline STAB2 & 1.5959 & 10.8391 & -2.76382 & 0.05233 & DOWN \\
\hline USP44 & 1.8601 & 10.9929 & -2.56311 & 0.01399 & DOWN \\
\hline$N A C A D$ & 1.0641 & 4.8078 & -2.17569 & 0.00880 & DOWN \\
\hline$N K \times 2-1$ & 1.0262 & 10.0430 & -3.29079 & 0.07015 & DOWN \\
\hline ZFHX2 & 2.1674 & 9.6776 & -2.15869 & 0.12362 & DOWN \\
\hline MYCBPAP & 2.0527 & 9.0242 & -2.13624 & 0.01979 & DOWN \\
\hline TBR1 & 1.0277 & 7.6811 & -2.90187 & 0.02225 & DOWN \\
\hline ERMN & 1.7316 & 7.4747 & -2.10993 & 0.08230 & DOWN \\
\hline GATA4 & 2.6139 & 13.7017 & -2.39009 & 0.01979 & DOWN \\
\hline IL36RN & 1.4774 & 15.3358 & -3.37576 & 0.05024 & DOWN \\
\hline$L M X 1 B$ & 1.3683 & 5.9877 & -2.12967 & 0.10001 & DOWN \\
\hline TFAP2A & 6.1128 & 31.6387 & -2.37179 & 0.00387 & DOWN \\
\hline TRIM29 & 5.9229 & 47.7608 & -3.01146 & 0.00387 & DOWN \\
\hline POU2F3 & 1.2640 & 5.6271 & -2.15439 & 0.04654 & DOWN \\
\hline ARHGAP2O & 1.1767 & 6.8398 & -2.53925 & 0.05329 & DOWN \\
\hline DUOX1 & 1.2547 & 8.0837 & -2.68765 & 0.00387 & DOWN \\
\hline STRAG & 3.7422 & 17.6031 & -2.23388 & 0.00387 & DOWN \\
\hline CYP19A1 & 1.4324 & 10.4002 & -2.86016 & 0.00387 & DOWN \\
\hline SEMAGD & 7.2832 & 29.1548 & -2.00110 & 0.02756 & DOWN \\
\hline GCOM1 & 1.4337 & 12.1360 & -3.08144 & 0.00655 & DOWN \\
\hline TRIM54 & 2.8293 & 12.3398 & -2.12480 & 0.12319 & DOWN \\
\hline CILP & 1.3552 & 5.7343 & -2.08111 & 0.03431 & DOWN \\
\hline FGF5 & 1.0508 & 6.9476 & -2.72505 & 0.07587 & DOWN \\
\hline BMPR1B & 2.2124 & 10.4904 & -2.24539 & 0.06503 & DOWN \\
\hline$E G F$ & 9.8888 & 95.8034 & -3.27622 & 0.00387 & DOWN \\
\hline COL2A1 & 3.2159 & 28.8945 & -3.16749 & 0.02225 & DOWN \\
\hline INHBE & 1.2711 & 6.7488 & -2.40859 & 0.10296 & DOWN \\
\hline TMEM132B & 2.1504 & 12.9887 & -2.59459 & 0.04013 & DOWN \\
\hline SSTR1 & 1.2080 & 5.0159 & -2.05392 & 0.02953 & DOWN \\
\hline REM2 & 1.1855 & 5.3649 & -2.17803 & 0.18441 & DOWN \\
\hline TSSK4 & 1.3100 & 5.6949 & -2.12007 & 0.16215 & DOWN \\
\hline SLC24A4 & 2.0213 & 8.5046 & -2.07296 & 0.05548 & DOWN \\
\hline DUOXA1 & 2.0167 & 14.7525 & -2.87091 & 0.00387 & DOWN \\
\hline DUOX2 & 1.5116 & 6.5155 & -2.10778 & 0.13976 & DOWN \\
\hline DISP2 & 1.6165 & 23.4003 & -3.85556 & 0.04691 & DOWN \\
\hline
\end{tabular}

Table S4 (continued) 


\begin{tabular}{|c|c|c|c|c|c|}
\hline Gene name & Cancer_FPKM & Non-cancer_FPKM & $\log 2$ (fold change) & FDR & Trend \\
\hline CYP11A1 & 1.6835 & 10.6518 & -2.66160 & 0.01979 & DOWn \\
\hline ADAMTS17 & 3.4815 & 17.1040 & -2.29654 & 0.00387 & DOWn \\
\hline LMAN1L & 1.8280 & 8.1517 & -2.15685 & 0.15479 & DOWn \\
\hline NTRK3 & 5.6003 & 23.1753 & -2.04902 & 0.00387 & Down \\
\hline MYLK3 & 2.0101 & 14.2163 & -2.82220 & 0.02756 & DOWn \\
\hline ADAD2 & 1.3984 & 7.5160 & -2.42620 & 0.07941 & DOWn \\
\hline MYOCD & 1.6679 & 8.6995 & -2.38294 & 0.03737 & DOWn \\
\hline RASL10B & 2.1628 & 13.3325 & -2.62397 & 0.00387 & DOWn \\
\hline LRRC46 & 1.1009 & 4.5133 & -2.03557 & 0.15139 & DOWn \\
\hline GNAL & 2.4257 & 11.6784 & -2.26737 & 0.12166 & DOWn \\
\hline ADCYAP1 & 1.2738 & 5.3063 & -2.05862 & 0.16494 & DOWn \\
\hline SLC13A5 & 1.0771 & 7.2507 & -2.75090 & 0.00387 & DOWn \\
\hline MAPK4 & 4.0253 & 16.1400 & -2.00349 & 0.13870 & DOWn \\
\hline CBLN2 & 1.3881 & 14.8844 & -3.42267 & 0.00387 & DOWn \\
\hline PNMT & 1.0353 & 5.5386 & -2.41941 & 0.13596 & DOWn \\
\hline ARL5C & 1.1026 & 9.1422 & -3.05161 & 0.03573 & DOWn \\
\hline KLK3 & 1.7818 & 16.8417 & -3.24067 & 0.00655 & DOWn \\
\hline MMEL1 & 2.7267 & 15.5663 & -2.51322 & 0.05784 & DOWn \\
\hline C1orf222 & 1.0937 & 5.8727 & -2.42486 & 0.00880 & DOWn \\
\hline PRDM16 & 5.5053 & 24.0705 & -2.12838 & 0.04758 & DOWn \\
\hline FHAD1 & 2.9305 & 14.8649 & -2.34268 & 0.00387 & DOWn \\
\hline GPR161 & 3.9586 & 16.6552 & -2.07290 & 0.03205 & DOWN \\
\hline ADCY10 & 1.6242 & 8.9589 & -2.46359 & 0.19484 & DOWn \\
\hline SUSD4 & 5.7278 & 26.4717 & -2.20840 & 0.00387 & DOWn \\
\hline CRNN & 1.7262 & 12.2304 & -2.82480 & 0.16782 & DOWn \\
\hline$A Q P 10$ & 1.0819 & 4.6760 & -2.11165 & 0.17153 & DOWn \\
\hline$F L G$ & 1.1428 & 8.8859 & -2.95899 & 0.06125 & DOWN \\
\hline CNIH3 & 5.1366 & 21.1800 & -2.04382 & 0.05459 & DOWN \\
\hline OSR1 & 1.6861 & 13.1300 & -2.96109 & 0.03737 & DOWn \\
\hline REG3G & 1.9374 & 12.9047 & -2.73572 & 0.07979 & DOWN \\
\hline ANKRD53 & 1.3107 & 12.2109 & -3.21979 & 0.05503 & DOWN \\
\hline$D Q X 1$ & 1.8794 & 7.9892 & -2.08779 & 0.17123 & DOWN \\
\hline ST6GAL2 & 1.9272 & 10.5922 & -2.45845 & 0.02839 & DOWn \\
\hline CNGA3 & 1.0163 & 10.7642 & -3.40486 & 0.04379 & DOWN \\
\hline TMEFF2 & 1.2330 & 8.8040 & -2.83594 & 0.13576 & DOWN \\
\hline$D L X 1$ & 1.1416 & 12.7442 & -3.48065 & 0.00387 & DOWN \\
\hline UNC80 & 1.2158 & 8.1445 & -2.74392 & 0.00655 & DOWN \\
\hline TRPM8 & 1.2504 & 11.2201 & -3.16560 & 0.00387 & DOWN \\
\hline ESPNL & 1.3227 & 9.1291 & -2.78699 & 0.00880 & DOWN \\
\hline GADL1 & 1.6114 & 9.2660 & -2.52367 & 0.06153 & DOWN \\
\hline ACKR2 & 1.7010 & 12.6614 & -2.89600 & 0.00387 & DOWN \\
\hline FAM198A & 2.5663 & 13.5646 & -2.40210 & 0.02336 & DOWN \\
\hline SLC22A14 & 1.4825 & 8.2974 & -2.48464 & 0.06298 & DOWN \\
\hline TAGLN3 & 1.9045 & 11.6918 & -2.61804 & 0.03336 & DOWn \\
\hline$N R 112$ & 1.1137 & 6.3257 & -2.50592 & 0.03336 & DOWN \\
\hline TMEM108 & 1.1791 & 14.0857 & -3.57853 & 0.00387 & DOWN \\
\hline MUC4 & 2.0909 & 13.0009 & -2.63641 & 0.00387 & DOWN \\
\hline ECE2 & 1.7953 & 9.7745 & -2.44478 & 0.10717 & DOWN \\
\hline VWA5B2 & 1.6520 & 11.9582 & -2.85570 & 0.05924 & DOWN \\
\hline RNF175 & 1.3105 & 9.5195 & -2.86083 & 0.01979 & DOWN \\
\hline $\mathrm{CDH} 18$ & 2.7142 & 14.8307 & -2.45000 & 0.02839 & DOWN \\
\hline GABRB2 & 1.3512 & 6.1523 & -2.18693 & 0.06727 & DOWN \\
\hline CPLX2 & 1.8329 & 14.6904 & -3.00271 & 0.00387 & DOWN \\
\hline MLIP & 1.3868 & 17.7006 & -3.67399 & 0.00387 & DOWN \\
\hline FAXC & 1.0252 & 4.2803 & -2.06180 & 0.07426 & DOWN \\
\hline TRIM50 & 1.5346 & 10.9963 & -2.84104 & 0.08695 & DOWN \\
\hline$A S B 10$ & 1.0502 & 8.4709 & -3.01190 & 0.08295 & DOWn \\
\hline PXDNL & 1.2045 & 8.0889 & -2.74749 & 0.00880 & DOWn \\
\hline$S T 18$ & 2.5225 & 34.7950 & -3.78594 & 0.00387 & DOWn \\
\hline RSPO2 & 1.1349 & 8.5042 & -2.90563 & 0.01241 & DOWN \\
\hline FAM135B & 2.5607 & 13.2629 & -2.37280 & 0.00655 & DOWN \\
\hline CDKN2B & 8.5338 & 35.8029 & -2.06882 & 0.11588 & DOWn \\
\hline ACTL7B & 1.1751 & 7.9945 & -2.76620 & 0.01979 & DOWN \\
\hline$R G R$ & 1.2349 & 6.7846 & -2.45791 & 0.03857 & DOWN \\
\hline HTR7 & 1.1403 & 6.6041 & -2.53392 & 0.06393 & DOWN \\
\hline SYT8 & 1.9311 & 10.3322 & -2.41962 & 0.01560 & DOWN \\
\hline KLHL35 & 1.1152 & 4.8428 & -2.11849 & 0.14453 & DOWN \\
\hline DRD2 & 1.0163 & 9.6847 & -3.25244 & 0.00387 & DOWN \\
\hline GRIK4 & 1.6957 & 8.6911 & -2.35763 & 0.09163 & DOWN \\
\hline ADAM33 & 1.2617 & 7.6260 & -2.59551 & 0.05784 & DOWn \\
\hline KIRREL3 & 1.4454 & 9.7097 & -2.74791 & 0.00880 & DOWN \\
\hline FAM57B & 1.3186 & 7.6954 & -2.54496 & 0.14547 & DOWN \\
\hline HMGA2 & 1.9395 & 10.9656 & -2.49921 & 0.00387 & DOWn \\
\hline
\end{tabular}

Table S4 (continued) 


\begin{tabular}{|c|c|c|c|c|c|}
\hline Gene name & Cancer_FPKM & Non-cancer_FPKM & $\log 2$ (fold change) & FDR & Trend \\
\hline PCDH15 & 1.3704 & 7.0431 & -2.36159 & 0.00387 & DOWN \\
\hline $\mathrm{CDH} 8$ & 2.7232 & 14.5363 & -2.41628 & 0.00655 & DOWN \\
\hline LYPD6B & 1.8429 & 7.7467 & -2.07157 & 0.04379 & DOWN \\
\hline CNDP1 & 1.3604 & 5.8316 & -2.09987 & 0.11443 & DOWN \\
\hline CACNA2D4 & 7.0745 & 29.6657 & -2.06810 & 0.00387 & DOWN \\
\hline MAT1A & 1.3854 & 6.4889 & -2.22767 & 0.17123 & DOWN \\
\hline AKR1C2 & 2.0456 & 10.2319 & -2.32245 & 0.01081 & DOWN \\
\hline DPYSL4 & 1.4058 & 14.7352 & -3.38984 & 0.05233 & DOWN \\
\hline GABRA2 & 2.2435 & 10.0330 & -2.16091 & 0.01241 & DOWN \\
\hline TMEM163 & 2.3896 & 10.7040 & -2.16328 & 0.18784 & DOWN \\
\hline RIT2 & 1.5442 & 12.0062 & -2.95886 & 0.15202 & DOWN \\
\hline$S H 2 D 6$ & 2.7231 & 11.0224 & -2.01713 & 0.10746 & DOWN \\
\hline TRIM36 & 3.2235 & 14.8853 & -2.20719 & 0.02225 & DOWN \\
\hline GRIA4 & 1.4076 & 7.0618 & -2.32679 & 0.00387 & DOWN \\
\hline TCTEX1D1 & 1.6870 & 14.2003 & -3.07341 & 0.06247 & DOWN \\
\hline HHEX & 5.0962 & 20.8967 & -2.03579 & 0.09947 & DOWN \\
\hline GRM1 & 1.9980 & 20.6552 & -3.36990 & 0.00387 & DOWN \\
\hline ZIC1 & 1.3839 & 11.7494 & -3.08582 & 0.03336 & DOWN \\
\hline$L G / 2$ & 1.5657 & 6.9949 & -2.15953 & 0.02109 & DOWN \\
\hline$A C O X L$ & 1.5742 & 9.9141 & -2.65485 & 0.00387 & DOWN \\
\hline PTPRR & 1.1400 & 5.0026 & -2.13360 & 0.08742 & DOWN \\
\hline SCN3A & 1.3961 & 6.8391 & -2.29238 & 0.04379 & DOWN \\
\hline PLEKHG4B & 1.1928 & 5.8255 & -2.28804 & 0.01560 & DOWN \\
\hline HSЗST3A1 & 3.6377 & 19.2828 & -2.40622 & 0.02439 & DOWN \\
\hline AK5 & 1.9971 & 9.6128 & -2.26708 & 0.00387 & DOWN \\
\hline CABYR & 1.7796 & 7.2546 & -2.02739 & 0.07175 & DOWN \\
\hline JPH3 & 1.1456 & 9.9048 & -3.11199 & 0.02648 & DOWN \\
\hline $\mathrm{CDH} 12$ & 1.0736 & 5.4910 & -2.35458 & 0.06906 & DOWN \\
\hline CERS3 & 1.5683 & 11.8336 & -2.91563 & 0.02648 & DOWN \\
\hline CHODL & 1.0773 & 4.5535 & -2.07962 & 0.03336 & DOWN \\
\hline PDE1C & 12.1834 & 49.7045 & -2.02847 & 0.00655 & DOWN \\
\hline EME1 & 2.0895 & 12.2115 & -2.54702 & 0.04141 & DOWN \\
\hline CNTNAP5 & 2.4244 & 13.2283 & -2.44794 & 0.11729 & DOWN \\
\hline GRIA1 & 1.4931 & 10.8842 & -2.86589 & 0.00387 & DOWN \\
\hline FMN2 & 2.1708 & 12.4225 & -2.51669 & 0.02648 & DOWN \\
\hline GPR61 & 1.3359 & 9.5198 & -2.83309 & 0.02336 & DOWN \\
\hline CLDN8 & 4.2627 & 23.5310 & -2.46471 & 0.02439 & DOWN \\
\hline$P P P 2 R 2 B$ & 3.5623 & 14.9878 & -2.07292 & 0.00387 & DOWN \\
\hline UNC5D & 1.9130 & 8.1266 & -2.08679 & 0.11052 & DOWN \\
\hline GAREML & 2.1216 & 10.2975 & -2.27909 & 0.12182 & DOWN \\
\hline RADIL & 1.0107 & 8.3482 & -3.04610 & 0.01712 & DOWN \\
\hline EXTL1 & 1.2214 & 8.5849 & -2.81331 & 0.09896 & DOWN \\
\hline ESYT3 & 2.7009 & 13.2869 & -2.29848 & 0.01712 & DOWN \\
\hline C2orf62 & 2.8931 & 12.4324 & -2.10344 & 0.111111 & DOWN \\
\hline CD1E & 1.3944 & 6.5149 & -2.22412 & 0.00655 & DOWN \\
\hline PLA2G2F & 1.4016 & 10.0959 & -2.84863 & 0.09632 & DOWN \\
\hline FGF17 & 1.4966 & 8.9430 & -2.57906 & 0.08011 & DOWN \\
\hline ACTC1 & 1.2520 & 5.0388 & -2.00883 & 0.17903 & DOWN \\
\hline SCUBE1 & 2.2452 & 12.2665 & -2.44981 & 0.00387 & DOWN \\
\hline IRX6 & 1.4938 & 9.6994 & -2.69894 & 0.03205 & DOWN \\
\hline CELF3 & 1.0083 & 8.4344 & -3.06436 & 0.03857 & DOWN \\
\hline RGL4 & 1.6133 & 11.7842 & -2.86881 & 0.05960 & DOWN \\
\hline FAM131B & 1.2662 & 10.1259 & -2.99945 & 0.00387 & DOWN \\
\hline FNDC5 & 1.2590 & 16.3353 & -3.69764 & 0.00880 & DOWN \\
\hline TMPRSS3 & 2.4442 & 11.6124 & -2.24823 & 0.02839 & DOWN \\
\hline FCRL3 & 2.8201 & 12.9180 & -2.19554 & 0.03281 & DOWN \\
\hline LYGK & 1.6708 & 11.0794 & -2.72923 & 0.04013 & DOWN \\
\hline RECQL4 & 3.5721 & 15.7277 & -2.13849 & 0.02336 & DOWN \\
\hline NPHS1 & 4.7907 & 19.9849 & -2.06060 & 0.18285 & DOWN \\
\hline GRIN2C & 1.1117 & 8.5862 & -2.94922 & 0.00880 & DOWN \\
\hline$C C D C 155$ & 3.8395 & 26.7289 & -2.79940 & 0.01560 & DOWN \\
\hline CD300LG & 1.6037 & 15.1192 & -3.23687 & 0.02109 & DOWN \\
\hline SHANK1 & 1.1576 & 14.9095 & -3.68706 & 0.00655 & DOWN \\
\hline TREML1 & 1.0299 & 7.7564 & -2.91285 & 0.06393 & DOWN \\
\hline RNASEK-C17orf49 & 1.3188 & 8.0372 & -2.60743 & 0.16202 & DOWN \\
\hline CCDC78 & 1.5015 & 6.5305 & -2.12078 & 0.02537 & DOWN \\
\hline C16orf59 & 1.9151 & 13.8582 & -2.85528 & 0.00655 & DOWN \\
\hline ZG16B & 1.6312 & 11.9623 & -2.87446 & 0.03939 & DOWN \\
\hline ITIH3 & 4.3097 & 25.4460 & -2.56179 & 0.00880 & DOWN \\
\hline CYP4A22 & 1.6425 & 8.9790 & -2.45071 & 0.12062 & DOWN \\
\hline SLC1A7 & 1.5262 & 10.1497 & -2.73341 & 0.15362 & DOWN \\
\hline KNCN & 1.2904 & 10.6202 & -3.04091 & 0.06594 & DOWN \\
\hline PDPN & 2.1410 & 8.8707 & -2.05075 & 0.04654 & DOWN \\
\hline
\end{tabular}




\begin{tabular}{|c|c|c|c|c|c|}
\hline Gene name & Cancer_FPKM & Non-cancer_FPKM & $\log 2$ (fold change) & FDR & Trend \\
\hline MATN1 & 1.4755 & 8.0907 & -2.45511 & 0.09977 & DOWn \\
\hline WNT4 & 2.0055 & 15.2043 & -2.92245 & 0.07468 & DOWn \\
\hline FCGR3B & 1.9261 & 9.3866 & -2.28491 & 0.12985 & DOWn \\
\hline AXDND1 & 2.1052 & 10.5149 & -2.32044 & 0.17297 & Down \\
\hline KLHDC8A & 3.9796 & 19.1271 & -2.26494 & 0.02839 & DOWn \\
\hline C1orf145 & 1.4398 & 5.8743 & -2.02854 & 0.11921 & DOWn \\
\hline LRRTM1 & 2.3003 & 9.6762 & -2.07262 & 0.19084 & DOWn \\
\hline KCNF1 & 1.5538 & 9.1001 & -2.55008 & 0.00387 & DOWn \\
\hline MSX1 & 2.1096 & 9.2171 & -2.12732 & 0.03797 & DOWn \\
\hline NIPAL1 & 3.5056 & 18.9018 & -2.43081 & 0.14841 & DOWn \\
\hline SPTA1 & 2.1646 & 18.9242 & -3.12804 & 0.02225 & DOWn \\
\hline EFHB & 1.3828 & 9.6010 & -2.79554 & 0.07401 & DOWn \\
\hline CADPS & 2.6169 & 36.1534 & -3.78822 & 0.00387 & DOWn \\
\hline PRRT3 & 1.3919 & 6.2608 & -2.16927 & 0.11443 & DOWn \\
\hline$C X C L 3$ & 2.5405 & 11.2573 & -2.14771 & 0.18335 & DOWn \\
\hline TGM4 & 1.6454 & 13.7988 & -3.06801 & 0.02756 & DOWn \\
\hline GRIK3 & 4.0564 & 17.0625 & -2.07256 & 0.05819 & DOWn \\
\hline MFI2 & 4.9025 & 25.0917 & -2.35563 & 0.00655 & DOWn \\
\hline CDC25A & 1.6653 & 8.5203 & -2.35516 & 0.02109 & DOWn \\
\hline$B S N$ & 1.0191 & 9.1517 & -3.16681 & 0.00387 & DOWn \\
\hline CAMKV & 1.1050 & 13.6353 & -3.62525 & 0.00387 & DOWn \\
\hline MST1R & 2.3718 & 10.9886 & -2.21197 & 0.04691 & DOWN \\
\hline GRM2 & 1.0613 & 10.2071 & -3.26563 & 0.00387 & DOWn \\
\hline PITX2 & 4.0647 & 26.7912 & -2.72053 & 0.01847 & DOWn \\
\hline HAND2 & 1.8211 & 14.3861 & -2.98179 & 0.08467 & DOWn \\
\hline TMEM155 & 1.3588 & 6.5494 & -2.26906 & 0.06153 & DOWn \\
\hline RANBP3L & 4.2740 & 22.3698 & -2.38788 & 0.17549 & DOWN \\
\hline GPR98 & 3.4751 & 14.4260 & -2.05353 & 0.07426 & DOWn \\
\hline C5orf63 & 4.3913 & 19.7241 & -2.16725 & 0.02648 & DOWn \\
\hline HTR4 & 1.4608 & 14.5327 & -3.31448 & 0.00387 & DOWn \\
\hline TIGD6 & 3.2856 & 13.2230 & -2.00880 & 0.14943 & DOWn \\
\hline TERT & 1.3342 & 5.5405 & -2.05409 & 0.19590 & DOWn \\
\hline ACSL6 & 4.2795 & 25.2395 & -2.56018 & 0.00387 & DOWn \\
\hline RAET1E & 1.0594 & 4.9446 & -2.22264 & 0.03737 & DOWn \\
\hline MYOZ3 & 1.9292 & 16.3702 & -3.08501 & 0.00387 & DOWn \\
\hline KIF6 & 1.5083 & 6.9385 & -2.20173 & 0.04889 & DOWn \\
\hline TMEM74 & 1.2513 & 10.6958 & -3.09556 & 0.11138 & DOWN \\
\hline OSR2 & 2.1348 & 15.0057 & -2.81336 & 0.01847 & DOWn \\
\hline BAALC & 1.2758 & 10.4114 & -3.02873 & 0.00655 & DOWn \\
\hline DCSTAMP & 1.5551 & 13.6720 & -3.13619 & 0.03573 & DOWn \\
\hline C9orf24 & 1.2131 & 5.2559 & -2.11521 & 0.09505 & DOWn \\
\hline ZMAT4 & 2.0473 & 9.9057 & -2.27455 & 0.05960 & DOWn \\
\hline C8orf34 & 1.6779 & 8.9162 & -2.40978 & 0.00880 & DOWn \\
\hline TRPV6 & 3.5452 & 16.2657 & -2.19788 & 0.01560 & DOWn \\
\hline C7orf34 & 1.4653 & 7.7891 & -2.41032 & 0.18663 & DOWn \\
\hline WNK2 & 4.2902 & 24.2634 & -2.49968 & 0.00387 & DOWn \\
\hline SLITRK5 & 1.6962 & 7.7627 & -2.19427 & 0.08194 & DOWn \\
\hline FAT3 & 3.1207 & 15.3502 & -2.29833 & 0.00655 & DOWn \\
\hline LRFN5 & 1.7496 & 20.2469 & -3.53263 & 0.01241 & DOWn \\
\hline ZNF488 & 2.4674 & 14.3617 & -2.54117 & 0.04187 & DOWn \\
\hline TSHR & 2.1019 & 9.9055 & -2.23651 & 0.07588 & DOWN \\
\hline TMEM63C & 1.3230 & 6.4443 & -2.28418 & 0.10588 & DOWn \\
\hline AMER2 & 1.0689 & 9.6523 & -3.17472 & 0.05385 & DOWn \\
\hline PPP1R36 & 1.6052 & 6.9752 & -2.11945 & 0.15171 & DOWn \\
\hline E2F7 & 1.3620 & 5.5400 & -2.02415 & 0.02336 & DOWn \\
\hline NELL1 & 3.6122 & 17.6026 & -2.28484 & 0.05879 & DOWn \\
\hline C1QL3 & 1.1942 & 5.7844 & -2.27607 & 0.00655 & DOWn \\
\hline KCNC2 & 2.0068 & 12.6553 & -2.65674 & 0.03737 & Down \\
\hline CMTM5 & 1.5715 & 12.9596 & -3.04380 & 0.02336 & DOWn \\
\hline GLB1L3 & 1.4587 & 12.3495 & -3.08172 & 0.00880 & DOWn \\
\hline ADAMTS15 & 2.9024 & 12.2789 & -2.08088 & 0.00387 & DOWn \\
\hline LRTM2 & 2.0096 & 9.2701 & -2.20567 & 0.08324 & DOWn \\
\hline TMEM100 & 1.0870 & 5.8643 & -2.43165 & 0.06954 & Down \\
\hline NETO1 & 1.8968 & 13.7028 & -2.85284 & 0.03857 & DOWn \\
\hline CYB5R2 & 2.8021 & 27.2247 & -3.28034 & 0.00387 & DOWn \\
\hline SERPINBT & 1.0036 & 4.9096 & -2.29041 & 0.07979 & DOWn \\
\hline BEAN1 & 2.1149 & 15.8687 & -2.90752 & 0.00387 & DOWn \\
\hline CPLX4 & 1.2655 & 5.3562 & -2.08153 & 0.18026 & DOWn \\
\hline $\mathrm{ZCCHC18}$ & 1.9948 & 8.1566 & -2.03172 & 0.11371 & DOWn \\
\hline YPEL4 & 1.9571 & 8.0327 & -2.03720 & 0.11225 & DOWn \\
\hline PLIN1 & 3.0015 & 12.9594 & -2.11022 & 0.17451 & DOWN \\
\hline GREM1 & 1.6957 & 9.7799 & -2.52791 & 0.06153 & DOWn \\
\hline MS4A14 & 1.8211 & 7.6889 & -2.07799 & 0.19970 & DOWn \\
\hline
\end{tabular}




\begin{tabular}{|c|c|c|c|c|c|}
\hline Gene name & Cancer_FPKM & Non-cancer_FPKM & log2(fold change) & FDR & Trend \\
\hline EPB42 & 1.1762 & 7.9643 & -2.75947 & 0.16802 & DOWN \\
\hline TCP10L2 & 1.2342 & 8.7963 & -2.83338 & 0.01979 & DOWN \\
\hline TMEM92 & 1.6376 & 7.4292 & -2.18165 & 0.01712 & DOWN \\
\hline ISLR2 & 2.2529 & 10.4272 & -2.21051 & 0.01979 & DOWN \\
\hline LOXHD1 & 1.8385 & 13.5908 & -2.88605 & 0.00387 & DOWN \\
\hline IGF2 & 1.5055 & 8.7953 & -2.54646 & 0.14461 & DOWN \\
\hline RBFOX3 & 2.6353 & 30.4001 & -3.52803 & 0.00387 & DOWN \\
\hline PROCA1 & 3.6641 & 15.1827 & -2.05090 & 0.00387 & DOWN \\
\hline CORO6 & 2.2124 & 9.6548 & -2.12564 & 0.05960 & DOWN \\
\hline$A Q P 2$ & 34.4014 & 314.3140 & -3.19167 & 0.19405 & DOWN \\
\hline ANKRD33 & 1.1989 & 7.8403 & -2.70916 & 0.02953 & DOWN \\
\hline TTYH1 & 1.7639 & 7.8649 & -2.15665 & 0.00387 & DOWN \\
\hline$P S C A$ & 2.3018 & 15.3477 & -2.73719 & 0.07047 & DOWN \\
\hline TRPV3 & 1.2606 & 8.7779 & -2.79975 & 0.05960 & DOWN \\
\hline KLK1 & 1.1769 & 10.1195 & -3.10409 & 0.05924 & DOWN \\
\hline KLK2 & 2.8110 & 25.1975 & -3.16415 & 0.00387 & DOWN \\
\hline KLK11 & 1.1479 & 8.1591 & -2.82935 & 0.03939 & DOWN \\
\hline MGAT5B & 1.6848 & 10.5701 & -2.64930 & 0.01081 & DOWN \\
\hline SCARA3 & 3.6710 & 14.6887 & -2.00045 & 0.02439 & DOWN \\
\hline SCARA5 & 1.7970 & 7.4509 & -2.05186 & 0.17751 & DOWN \\
\hline PNOC & 1.7439 & 8.0888 & -2.21361 & 0.10990 & DOWN \\
\hline$H R$ & 3.0898 & 15.6785 & -2.34323 & 0.03336 & DOWN \\
\hline SFTPC & 1.0818 & 8.4815 & -2.97083 & 0.01847 & DOWN \\
\hline PHYHIP & 1.9280 & 15.4350 & -3.00107 & 0.00387 & DOWN \\
\hline CHRM1 & 1.2075 & 7.3837 & -2.61230 & 0.11820 & DOWN \\
\hline GDNF & 1.0794 & 22.1578 & -4.35951 & 0.00387 & DOWN \\
\hline VWA3B & 1.2658 & 8.2473 & -2.70392 & 0.00387 & DOWN \\
\hline KCTD19 & 1.4027 & 6.3071 & -2.16879 & 0.08504 & DOWN \\
\hline LRP1B & 1.4032 & 5.9362 & -2.08086 & 0.08467 & DOWN \\
\hline$C X X C 4$ & 1.7086 & 7.1277 & -2.06067 & 0.09137 & DOWN \\
\hline SHOX2 & 1.4123 & 9.4855 & -2.74767 & 0.08742 & DOWN \\
\hline АТОН8 & 4.3953 & 19.5524 & -2.15331 & 0.01560 & DOWN \\
\hline STXBPG & 4.0774 & 20.0143 & -2.29530 & 0.03042 & DOWN \\
\hline GRM5 & 1.7480 & 11.2137 & -2.68147 & 0.03135 & DOWN \\
\hline IL13 & 1.7191 & 12.2524 & -2.83332 & 0.12143 & DOWN \\
\hline SH3TC2 & 2.6061 & 15.0426 & -2.52911 & 0.00387 & DOWN \\
\hline C22orf15 & 1.0505 & 10.0300 & -3.25512 & 0.10971 & DOWN \\
\hline UMOD & 322.0180 & $1,581.1700$ & -2.29578 & 0.10074 & DOWN \\
\hline GP2 & 6.2821 & 27.1751 & -2.11296 & 0.12404 & DOWN \\
\hline CTNND2 & 1.1562 & 6.9836 & -2.59464 & 0.00387 & DOWN \\
\hline KCNAB3 & 2.0300 & 9.9246 & -2.28955 & 0.06125 & DOWN \\
\hline NSG2 & 1.1870 & 7.2905 & -2.61865 & 0.00655 & DOWN \\
\hline SERPINAG & 3.2618 & 17.6907 & -2.43926 & 0.05233 & DOWN \\
\hline FOXD4 & 1.4778 & 9.3829 & -2.66657 & 0.00655 & DOWN \\
\hline MRGPRX1 & 1.4045 & 10.0331 & -2.83665 & 0.17521 & DOWN \\
\hline SEMA3E & 2.0254 & 9.9708 & -2.29952 & 0.16392 & DOWN \\
\hline VSTM2A & 1.4024 & 20.0056 & -3.83439 & 0.02336 & DOWN \\
\hline$S G C D$ & 1.4361 & 5.8962 & -2.03765 & 0.01560 & DOWN \\
\hline GPR37 & 1.8776 & 9.0453 & -2.26829 & 0.01399 & DOWN \\
\hline PSG6 & 3.3034 & 20.6101 & -2.64133 & 0.00387 & DOWN \\
\hline CEACAM3 & 2.3413 & 16.0010 & -2.77277 & 0.02953 & DOWN \\
\hline FPR2 & 1.7499 & 7.2168 & -2.04408 & 0.18356 & DOWN \\
\hline C8orf74 & 1.0361 & 11.7999 & -3.50950 & 0.00655 & DOWN \\
\hline$A L K$ & 1.9375 & 13.7053 & -2.82250 & 0.04090 & DOWN \\
\hline PROL1 & 1.0237 & 8.7535 & -3.09603 & 0.08828 & DOWN \\
\hline NPTX1 & 2.0137 & 8.7358 & -2.11706 & 0.08194 & DOWN \\
\hline KRT15 & 1.4240 & 10.9587 & -2.94411 & 0.00387 & DOWN \\
\hline KRT13 & 3.3453 & 24.1010 & -2.84888 & 0.03737 & DOWN \\
\hline KSR2 & 1.4306 & 10.6844 & -2.90081 & 0.00387 & DOWN \\
\hline COL24A1 & 1.3502 & 8.8503 & -2.71255 & 0.03281 & DOWN \\
\hline PCDHB1 & 1.0385 & 7.9833 & -2.94250 & 0.01847 & DOWN \\
\hline NINJ2 & 2.3774 & 11.1801 & -2.23346 & 0.08595 & DOWN \\
\hline LGALS9C & 1.3688 & 8.0950 & -2.56410 & 0.05879 & DOWN \\
\hline FOXB1 & 1.8784 & 15.5277 & -3.04729 & 0.12859 & DOWN \\
\hline REG3A & 1.2930 & 19.2338 & -3.89483 & 0.01241 & DOWN \\
\hline REG1B & 1.2173 & 6.4894 & -2.41443 & 0.05548 & DOWN \\
\hline ABCG4 & 2.4646 & 17.9363 & -2.86349 & 0.05710 & DOWN \\
\hline PRSS27 & 2.0389 & 9.4991 & -2.22001 & 0.07175 & DOWN \\
\hline C2orf54 & 2.1172 & 10.9529 & -2.37105 & 0.13576 & DOWN \\
\hline$A G X T$ & 2.2997 & 16.4344 & -2.83720 & 0.02648 & DOWN \\
\hline CARNS1 & 1.0983 & 6.3471 & -2.53084 & 0.08790 & DOWN \\
\hline$S N \times 32$ & 1.7688 & 13.2596 & -2.90619 & 0.02756 & DOWN \\
\hline OVOL1 & 1.9558 & 11.6182 & -2.57058 & 0.04437 & DOWN \\
\hline
\end{tabular}


Table S4 (continued)

\begin{tabular}{|c|c|c|c|c|c|}
\hline Gene name & Cancer_FPKM & Non-cancer_FPKM & $\log 2$ (fold change) & FDR & Trend \\
\hline AQPEP & 1.3292 & 8.4646 & -2.67093 & 0.04691 & DOWN \\
\hline MRGPRF & 2.9806 & 18.5720 & -2.63948 & 0.02537 & DOWN \\
\hline ARPP21 & 1.8205 & 18.7284 & -3.36279 & 0.00387 & DOWN \\
\hline RP11-683L23.1 & 1.2271 & 15.0884 & -3.62014 & 0.03737 & DOWN \\
\hline SLC2A14 & 1.8558 & 10.1889 & -2.45688 & 0.00655 & DOWN \\
\hline$D A B 1$ & 1.1783 & 9.7049 & -3.04196 & 0.00387 & DOWN \\
\hline CCDC36 & 4.0788 & 18.7375 & -2.19971 & 0.14532 & DOWN \\
\hline C2orf70 & 1.3119 & 7.4850 & -2.51234 & 0.11750 & DOWN \\
\hline GPR113 & 1.1564 & 6.9420 & -2.58577 & 0.11389 & DOWN \\
\hline XCR1 & 1.7973 & 7.9867 & -2.15179 & 0.10867 & DOWN \\
\hline HSPB7 & 2.2056 & 12.5944 & -2.51354 & 0.09797 & DOWN \\
\hline PHOSPHO1 & 1.3907 & 5.6388 & -2.01958 & 0.09551 & DOWN \\
\hline TUBB8 & 1.2534 & 8.4242 & -2.74868 & 0.07047 & DOWN \\
\hline CBX2 & 3.4815 & 16.4630 & -2.24143 & 0.00655 & DOWN \\
\hline C9orf131 & 1.7134 & 11.1189 & -2.69807 & 0.03205 & DOWN \\
\hline SLC16A11 & 1.8512 & 9.1467 & -2.30481 & 0.11778 & DOWN \\
\hline GALNTL6 & 2.1232 & 14.7362 & -2.79506 & 0.03042 & DOWN \\
\hline IGDCC3 & 1.1649 & 5.0227 & -2.10824 & 0.14787 & DOWN \\
\hline SLC26A9 & 2.2187 & 9.2199 & -2.05506 & 0.03939 & DOWN \\
\hline MFSD4 & 11.0637 & 57.6549 & -2.38161 & 0.04585 & DOWN \\
\hline TMEM81 & 2.2897 & 9.3406 & -2.02837 & 0.02336 & DOWN \\
\hline BRSK2 & 1.1687 & 7.0507 & -2.59289 & 0.00387 & DOWN \\
\hline$A M Z 1$ & 1.4375 & 6.8645 & -2.25557 & 0.16933 & DOWN \\
\hline ZIC4 & 1.5568 & 24.3535 & -3.96748 & 0.00387 & DOWN \\
\hline DES & 5.5612 & 61.8504 & -3.47530 & 0.16766 & DOWN \\
\hline SPSB4 & 2.2703 & 21.7403 & -3.25942 & 0.01081 & DOWN \\
\hline RAG2 & 1.6527 & 17.8718 & -3.43481 & 0.00387 & DOWN \\
\hline CADM2 & 1.2702 & 7.5653 & -2.57436 & 0.12166 & DOWN \\
\hline FAM182B & 3.4270 & 22.6674 & -2.72561 & 0.09524 & DOWN \\
\hline GAL3ST3 & 2.2305 & 10.7924 & -2.27461 & 0.19405 & DOWN \\
\hline GRAMD2 & 2.6725 & 11.0085 & -2.04235 & 0.00387 & DOWN \\
\hline CHRNA7 & 1.2036 & 6.6043 & -2.45604 & 0.14581 & DOWN \\
\hline SCUBE2 & 1.8694 & 9.3307 & -2.31938 & 0.00387 & DOWN \\
\hline UBQLNL & 1.7561 & 9.8823 & -2.49247 & 0.15231 & DOWN \\
\hline UBQLN3 & 2.1374 & 14.3828 & -2.75044 & 0.09505 & DOWN \\
\hline P2RY2 & 1.9212 & 8.6559 & -2.17169 & 0.07662 & DOWN \\
\hline GPR156 & 1.2504 & 9.7542 & -2.96360 & 0.00387 & DOWN \\
\hline EIF4E1B & 1.0420 & 7.6232 & -2.87105 & 0.03281 & DOWN \\
\hline DOK7 & 3.6909 & 22.3180 & -2.59616 & 0.02225 & DOWN \\
\hline PLEKHD1 & 1.2216 & 6.0927 & -2.31826 & 0.07047 & DOWN \\
\hline AMIGO3 & 1.3284 & 6.0563 & -2.18879 & 0.18898 & DOWN \\
\hline AIM1L & 1.8656 & 15.0620 & -3.01317 & 0.00387 & DOWN \\
\hline LRRTM4 & 1.2171 & 7.6152 & -2.64539 & 0.02336 & DOWN \\
\hline OR51B6 & 1.2648 & 12.1611 & -3.26532 & 0.13406 & DOWN \\
\hline OR4K13 & 1.0587 & 11.5990 & -3.45365 & 0.17521 & DOWN \\
\hline OR4N2 & 3.9325 & 28.6419 & -2.86460 & 0.18898 & DOWN \\
\hline AC110781.3 & 1.0994 & 8.1111 & -2.88322 & 0.13175 & DOWN \\
\hline DIRAS1 & 1.6990 & 7.9708 & -2.23002 & 0.16538 & DOWN \\
\hline GRIN1 & 1.0131 & 12.2730 & -3.59859 & 0.00387 & DOWN \\
\hline sox11 & 1.1683 & 5.0523 & -2.11256 & 0.05710 & DOWN \\
\hline FUT2 & 1.2230 & 6.4629 & -2.40177 & 0.11528 & DOWN \\
\hline LYGH & 1.1356 & 6.2145 & -2.45223 & 0.11417 & DOWN \\
\hline CLVS1 & 2.3005 & 22.7880 & -3.30824 & 0.02839 & DOWN \\
\hline FBХOЗ39 & 1.0596 & 4.9721 & -2.23030 & 0.15494 & DOWN \\
\hline C10orf71 & 1.5094 & 11.3935 & -2.91613 & 0.00387 & DOWN \\
\hline NIM1K & 1.6495 & 8.9009 & -2.43188 & 0.08718 & DOWN \\
\hline OR2G2 & 1.1885 & 7.9495 & -2.74175 & 0.19116 & DOWN \\
\hline NHLH2 & 1.0085 & 6.1672 & -2.61241 & 0.02336 & DOWN \\
\hline C12orf68 & 1.4851 & 7.1343 & -2.26420 & 0.00880 & DOWN \\
\hline PRSS42 & 2.2940 & 11.7986 & -2.36267 & 0.04966 & DOWN \\
\hline CA8 & 1.4914 & 14.1672 & -3.24781 & 0.00655 & DOWN \\
\hline FAM132B & 3.1436 & 13.4003 & -2.09177 & 0.03737 & DOWN \\
\hline CD300LB & 1.4374 & 7.6091 & -2.40425 & 0.05819 & DOWN \\
\hline TRIML2 & 1.2696 & 10.1401 & -2.99761 & 0.00387 & DOWN \\
\hline C12orf42 & 2.2124 & 9.1356 & -2.04586 & 0.01712 & DOWN \\
\hline GGN & 2.0205 & 12.9871 & -2.68433 & 0.04829 & DOWN \\
\hline C17orf96 & 1.1737 & 6.1578 & -2.39132 & 0.02439 & DOWN \\
\hline GATA2 & 6.4189 & 33.2709 & -2.37388 & 0.03493 & DOWN \\
\hline MKRN3 & 1.5590 & 11.1192 & -2.83434 & 0.00387 & DOWN \\
\hline LBX2 & 1.6758 & 7.1746 & -2.09805 & 0.11820 & DOWN \\
\hline TPPP2 & 1.1848 & 6.7381 & -2.50772 & 0.04141 & DOWN \\
\hline LRRC3B & 1.6815 & 13.1190 & -2.96383 & 0.04141 & DOWN \\
\hline MRGPRX3 & 1.0861 & 9.9607 & -3.19713 & 0.12207 & DOWN \\
\hline
\end{tabular}




\begin{tabular}{|c|c|c|c|c|c|}
\hline Gene name & Cancer_FPKM & Non-cancer_FPKM & log2(fold change) & FDR & Trend \\
\hline ITLN1 & 1.1105 & 5.9767 & -2.42809 & 0.14270 & DOWN \\
\hline NRXN1 & 3.4001 & 22.3314 & -2.71544 & 0.00387 & DOWN \\
\hline GPBAR1 & 1.2928 & 10.7516 & -3.05595 & 0.06153 & DOWN \\
\hline$T H$ & 2.2495 & 15.3266 & -2.76836 & 0.02537 & DOWN \\
\hline SLC9A4 & 2.6837 & 13.3339 & -2.31284 & 0.03281 & DOWN \\
\hline PLD5 & 1.6598 & 11.8960 & -2.84143 & 0.02336 & DOWN \\
\hline KCNE1 & 2.8083 & 14.1199 & -2.32998 & 0.02336 & DOWN \\
\hline PRR26 & 2.1317 & 8.8073 & -2.04672 & 0.16260 & DOWN \\
\hline RNF182 & 2.2348 & 11.4437 & -2.35632 & 0.07886 & DOWN \\
\hline GSX2 & 1.7144 & 9.0542 & -2.40087 & 0.14668 & DOWN \\
\hline CXCR2 & 1.0059 & 9.5426 & -3.24586 & 0.01081 & DOWN \\
\hline CHRM2 & 1.7820 & 18.8644 & -3.40407 & 0.00387 & DOWN \\
\hline PENK & 2.0170 & 20.6810 & -3.35801 & 0.00387 & DOWN \\
\hline TMEM132C & 1.1870 & 5.7916 & -2.28667 & 0.08790 & DOWN \\
\hline CCDC108 & 2.1087 & 11.1786 & -2.40633 & 0.04236 & DOWN \\
\hline sox 2 & 1.4039 & 9.5569 & -2.76708 & 0.17049 & DOWN \\
\hline OR6T1 & 1.1590 & 14.6323 & -3.65819 & 0.14980 & DOWN \\
\hline $\mathrm{OR} 52 \mathrm{H} 1$ & 1.1320 & 6.8029 & -2.58732 & 0.12319 & DOWN \\
\hline ATG9B & 3.0314 & 14.8863 & -2.29595 & 0.03573 & DOWN \\
\hline $\mathrm{OR} 8 \mathrm{H} 2$ & 1.0857 & 5.9950 & -2.46516 & 0.19857 & DOWN \\
\hline BAl1 & 1.8399 & 7.8108 & -2.08581 & 0.12143 & DOWN \\
\hline FTMT & 1.0155 & 6.4860 & -2.67514 & 0.19213 & DOWN \\
\hline RGMA & 2.5927 & 18.6495 & -2.84660 & 0.00387 & DOWN \\
\hline HHIPL1 & 1.0056 & 5.3393 & -2.40866 & 0.01081 & DOWN \\
\hline GABRG3 & 1.2511 & 7.3951 & -2.56339 & 0.03939 & DOWN \\
\hline KCNK4 & 1.0932 & 10.1171 & -3.21013 & 0.02109 & DOWN \\
\hline $\mathrm{NXPH3}$ & 1.3061 & 8.5497 & -2.71061 & 0.09929 & DOWN \\
\hline OR10G7 & 1.1384 & 11.0636 & -3.28075 & 0.19484 & DOWN \\
\hline OR4Q3 & 1.2128 & 11.7693 & -3.27861 & 0.16782 & DOWN \\
\hline KCNB2 & 1.0361 & 8.5278 & -3.04100 & 0.02839 & DOWN \\
\hline BRICD5 & 6.0436 & 27.7794 & -2.20055 & 0.08904 & DOWN \\
\hline RGS6 & 2.5201 & 17.0502 & -2.75824 & 0.00387 & DOWN \\
\hline GRID1 & 1.0636 & 6.7717 & -2.67063 & 0.14906 & DOWN \\
\hline GPR97 & 1.1178 & 6.4381 & -2.52602 & 0.03797 & DOWN \\
\hline SLC25A18 & 1.5111 & 6.2176 & -2.04079 & 0.08862 & DOWN \\
\hline C21orf90 & 1.1019 & 6.0760 & -2.46318 & 0.09481 & DOWN \\
\hline C19orf59 & 1.1668 & 7.7963 & -2.74022 & 0.11138 & DOWN \\
\hline BEGAIN & 2.0072 & 11.3704 & -2.50201 & 0.01241 & DOWN \\
\hline CSMD1 & 2.3762 & 12.5067 & -2.39600 & 0.00387 & DOWN \\
\hline $\mathrm{C} 2 \mathrm{CD} 4 \mathrm{C}$ & 1.3300 & 6.0671 & -2.18958 & 0.01241 & DOWN \\
\hline OR52L1 & 1.0449 & 8.5290 & -3.02896 & 0.16399 & DOWN \\
\hline SSTR3 & 1.2874 & 7.6012 & -2.56181 & 0.01979 & DOWN \\
\hline MEX3B & 1.8757 & 8.3602 & -2.15608 & 0.04654 & DOWN \\
\hline РСBP3 & 6.9221 & 34.0061 & -2.29651 & 0.00387 & DOWN \\
\hline CCR3 & 1.0621 & 14.4286 & -3.76389 & 0.00387 & DOWN \\
\hline MARCH11 & 2.5567 & 14.4958 & -2.50326 & 0.16228 & DOWN \\
\hline ASCL2 & 1.6185 & 7.0308 & -2.11907 & 0.04236 & DOWN \\
\hline TRAIP & 1.7204 & 9.3775 & -2.44648 & 0.01399 & DOWN \\
\hline AIFM3 & 3.3657 & 16.4446 & -2.28862 & 0.08230 & DOWN \\
\hline SCN5A & 1.0490 & 9.7146 & -3.21111 & 0.01081 & DOWN \\
\hline ARSI & 1.4029 & 8.4650 & -2.59305 & 0.03042 & DOWN \\
\hline$N P B$ & 1.3047 & 6.4250 & -2.30000 & 0.18129 & DOWN \\
\hline TMPRSS2 & 12.0821 & 49.1996 & -2.02577 & 0.06594 & DOWN \\
\hline TBX1 & 2.9917 & 13.1834 & -2.13970 & 0.16585 & DOWN \\
\hline CNTN2 & 1.5366 & 8.1021 & -2.39859 & 0.05767 & DOWN \\
\hline KCNJ12 & 1.7432 & 12.4800 & -2.83983 & 0.00880 & DOWN \\
\hline GOLGAGL4 & 1.3134 & 6.7130 & -2.35370 & 0.04585 & DOWN \\
\hline OLIG1 & 1.2981 & 5.4216 & -2.06231 & 0.13203 & DOWN \\
\hline TACSTD2 & 13.3267 & 57.1704 & -2.10095 & 0.01712 & DOWN \\
\hline KRTAP19-1 & 1.3714 & 11.3690 & -3.05142 & 0.18980 & DOWN \\
\hline PKP3 & 2.9119 & 15.8907 & -2.44814 & 0.04437 & DOWN \\
\hline C21orf88 & 1.4727 & 5.9452 & -2.01331 & 0.15192 & DOWN \\
\hline $\mathrm{ROBO} 2$ & 2.7206 & 13.8227 & -2.34505 & 0.06153 & DOWN \\
\hline NT5C1B & 1.3720 & 10.4913 & -2.93485 & 0.03042 & DOWN \\
\hline SLC24A3 & 1.4530 & 5.8704 & -2.01439 & 0.04013 & DOWN \\
\hline INPP5J & 4.4583 & 19.3608 & -2.11856 & 0.03939 & DOWN \\
\hline MFSD6L & 1.2327 & 6.8051 & -2.46485 & 0.00387 & DOWN \\
\hline SFTPA2 & 1.0339 & 8.7939 & -3.08846 & 0.05784 & DOWN \\
\hline SHC4 & 1.1732 & 8.3681 & -2.83450 & 0.03135 & DOWN \\
\hline NTF3 & 1.9387 & 11.8294 & -2.60926 & 0.04236 & DOWN \\
\hline SYN3 & 1.8961 & 17.9251 & -3.24089 & 0.11729 & DOWN \\
\hline ADARB2 & 2.5824 & 23.3592 & -3.17719 & 0.00387 & DOWN \\
\hline KCNQ5 & 1.6685 & 21.0549 & -3.65751 & 0.00880 & DOWN \\
\hline
\end{tabular}

Table S4 (continued) 


\begin{tabular}{|c|c|c|c|c|c|}
\hline Gene name & Cancer_FPKM & Non-cancer_FPKM & $\log 2$ (fold change) & FDR & Trend \\
\hline ADAMTSL5 & 2.1026 & 10.2644 & -2.28742 & 0.02109 & DOWn \\
\hline NATBL & 2.4407 & 13.8808 & -2.50773 & 0.02225 & DOWn \\
\hline TAS2R60 & 1.4757 & 10.3619 & -2.81186 & 0.18511 & DOWn \\
\hline KLHL34 & 1.0456 & 6.6467 & -2.66838 & 0.00387 & Down \\
\hline KRT5 & 3.7927 & 22.1922 & -2.54876 & 0.00655 & DOWn \\
\hline TEX38 & 1.1905 & 10.3122 & -3.11469 & 0.13394 & DOWn \\
\hline$K D M 4 D$ & 1.1826 & 5.7779 & -2.28856 & 0.02953 & DOWn \\
\hline GABRA5 & 1.2402 & 9.4133 & -2.92418 & 0.02109 & DOWn \\
\hline GLDN & 3.0892 & 18.0341 & -2.54541 & 0.00387 & DOWn \\
\hline MYT1L & 3.0669 & 31.2526 & -3.34913 & 0.00387 & DOWn \\
\hline C5orf38 & 2.6566 & 18.4211 & -2.79371 & 0.07543 & DOWn \\
\hline CLCNKA & 6.7145 & 55.5415 & -3.04822 & 0.02109 & DOWn \\
\hline OR9Q2 & 1.0552 & 10.8214 & -3.35832 & 0.19590 & DOWn \\
\hline FAM86B1 & 1.0040 & 5.3156 & -2.40441 & 0.11805 & DOWn \\
\hline MPPED1 & 1.8774 & 14.7388 & -2.97284 & 0.01081 & DOWn \\
\hline KRT16 & 1.5228 & 11.7834 & -2.95196 & 0.14980 & DOWn \\
\hline$Z A C N$ & 3.1807 & 12.8009 & -2.00883 & 0.16251 & DOWn \\
\hline PNLIPRP1 & 1.3514 & 8.0312 & -2.57120 & 0.00387 & DOWn \\
\hline LCE2A & 1.4268 & 18.8615 & -3.72461 & 0.12512 & DOWn \\
\hline$R P 11-195 F 19.5$ & 4.8957 & 22.6202 & -2.20802 & 0.12045 & DOWn \\
\hline MAGI2 & 4.0559 & 19.8056 & -2.28781 & 0.06557 & DOWn \\
\hline KCNJ11 & 1.7359 & 9.3888 & -2.43526 & 0.03042 & DOWN \\
\hline C1orf170 & 1.1533 & 5.6068 & -2.28137 & 0.18164 & DOWn \\
\hline DNAH17 & 1.2105 & 7.0580 & -2.54361 & 0.02537 & DOWn \\
\hline TTC24 & 1.6328 & 7.1148 & -2.12349 & 0.11443 & DOWn \\
\hline LCN10 & 2.2743 & 15.3972 & -2.75920 & 0.03797 & DOWn \\
\hline COL25A1 & 3.4429 & 23.8886 & -2.79464 & 0.05077 & DOWN \\
\hline SKOR1 & 1.1642 & 5.3976 & -2.21295 & 0.11956 & DOWn \\
\hline SHISAG & 1.5443 & 14.2848 & -3.20949 & 0.00387 & DOWn \\
\hline KLRG2 & 1.7199 & 16.6187 & -3.27243 & 0.14604 & DOWn \\
\hline GPR179 & 1.3001 & 6.4091 & -2.30149 & 0.05077 & DOWn \\
\hline MSANTD1 & 2.2561 & 12.8789 & -2.51311 & 0.04187 & DOWn \\
\hline KRT77 & 1.2158 & 7.7884 & -2.67936 & 0.13208 & DOWn \\
\hline FAM180A & 1.1002 & 9.1746 & -3.05985 & 0.05193 & DOWn \\
\hline S100A14 & 2.4532 & 11.2759 & -2.20053 & 0.17163 & DOWn \\
\hline FAM179A & 1.7811 & 8.2454 & -2.21079 & 0.09527 & DOWn \\
\hline TBC1D28 & 1.6264 & 8.9268 & -2.45649 & 0.03336 & DOWN \\
\hline PAX5 & 1.2856 & 7.6305 & -2.56936 & 0.00387 & DOWn \\
\hline KIF19 & 1.5426 & 7.9070 & -2.35780 & 0.10529 & DOWn \\
\hline CTSE & 1.3829 & 6.2008 & -2.16481 & 0.15824 & DOWn \\
\hline GREB1 & 3.0744 & 13.3222 & -2.11545 & 0.01560 & DOWn \\
\hline NOXO1 & 1.5005 & 6.3186 & -2.07415 & 0.16241 & DOWn \\
\hline FAM72A & 1.9547 & 15.8243 & -3.01716 & 0.00387 & DOWn \\
\hline SLC30A10 & 1.5309 & 10.6327 & -2.79605 & 0.03857 & DOWn \\
\hline SLC6A17 & 1.2404 & 7.5932 & -2.61391 & 0.00655 & DOWn \\
\hline$R P 11-366 L 20.2$ & 2.0139 & 12.1623 & -2.59438 & 0.05329 & DOWn \\
\hline FBXL22 & 1.6416 & 8.2167 & -2.32348 & 0.10466 & DOWn \\
\hline OR8S1 & 1.4798 & 11.7795 & -2.99279 & 0.09411 & Down \\
\hline GYPE & 1.0226 & 7.8264 & -2.93610 & 0.10971 & DOWn \\
\hline FAM177B & 1.5329 & 12.3834 & -3.01411 & 0.09855 & DOWn \\
\hline COLAAG & 2.3819 & 14.0278 & -2.55814 & 0.03042 & DOWN \\
\hline DLGAP2 & 2.0235 & 9.6370 & -2.25172 & 0.15529 & DOWN \\
\hline OR2T6 & 1.1627 & 20.0800 & -4.11027 & 0.13952 & DOWn \\
\hline$M B$ & 1.2400 & 7.5334 & -2.60293 & 0.04013 & DOWn \\
\hline ANTXRL & 1.1390 & 7.5966 & -2.73766 & 0.05924 & DOWn \\
\hline PEG3 & 3.4848 & 14.6126 & -2.06809 & 0.00387 & DOWn \\
\hline TMEM239 & 1.3876 & 6.3350 & -2.19078 & 0.18010 & DOWn \\
\hline DCAF12L2 & 1.0234 & 8.4256 & -3.04146 & 0.16976 & DOWn \\
\hline NTRK1 & 2.1268 & 9.1867 & -2.11083 & 0.16258 & DOWn \\
\hline$A R C$ & 1.2319 & 8.0989 & -2.71682 & 0.02756 & DOWn \\
\hline ZNF536 & 2.4901 & 16.3905 & -2.71859 & 0.03205 & DOWn \\
\hline RYR2 & 1.5535 & 8.3104 & -2.41936 & 0.03042 & DOWn \\
\hline TAT & 1.0287 & 8.5594 & -3.05666 & 0.04187 & Down \\
\hline$A B C A 4$ & 1.2329 & 15.5451 & -3.65632 & 0.00387 & DOWn \\
\hline MT-ND6 & 530.3260 & $22,334.5000$ & -5.39625 & 0.00387 & DOWn \\
\hline MT-CO2 & 636.0730 & $5,042.6300$ & -2.98691 & 0.03205 & DOWn \\
\hline MT-CYB & $1,043.8400$ & $14,942.2000$ & -3.83942 & 0.00387 & DOWn \\
\hline SMOC1 & 1.8984 & 8.9622 & -2.23910 & 0.08130 & DOWn \\
\hline COLGALT2 & 1.5137 & 7.7851 & -2.36260 & 0.04236 & DOWn \\
\hline EPS8L3 & 4.8180 & 32.7555 & -2.76524 & 0.07378 & DOWn \\
\hline MT-ND2 & $1,025.0800$ & $11,556.8000$ & -3.49493 & 0.01081 & DOWn \\
\hline APCDD1L & 1.8150 & 7.2664 & -2.00129 & 0.12116 & DOWn \\
\hline GRIN3A & 1.0027 & 9.1362 & -3.18776 & 0.00387 & DOWn \\
\hline
\end{tabular}

Table S4 (continued) 


\begin{tabular}{|c|c|c|c|c|c|}
\hline Gene name & Cancer_FPKM & Non-cancer_FPKM & log2(fold change) & FDR & Trend \\
\hline MT-ND5 & 730.9050 & $10,246.7000$ & -3.80933 & 0.00387 & DOWN \\
\hline BRINP2 & 1.1426 & 8.0815 & -2.82227 & 0.09527 & DOWN \\
\hline PAX9 & 2.7461 & 18.3078 & -2.73700 & 0.02439 & DOWN \\
\hline DUSP27 & 1.4671 & 8.7790 & -2.58109 & 0.02109 & DOWN \\
\hline CES1 & 1.9708 & 9.1446 & -2.21417 & 0.05077 & DOWN \\
\hline MT-ND4 & 39.4311 & $4,131.4700$ & -6.71118 & 0.12022 & DOWN \\
\hline MT-ND1 & 799.0490 & $6,561.9900$ & -3.03778 & 0.04187 & DOWN \\
\hline MT-ATP6 & 677.6510 & $7,392.9900$ & -3.44754 & 0.00880 & DOWN \\
\hline C1orf95 & 1.9609 & 8.4744 & -2.11159 & 0.00387 & DOWN \\
\hline TCP10 & 1.3872 & 12.9789 & -3.22596 & 0.00387 & DOWN \\
\hline CAPN8 & 2.3052 & 10.3725 & -2.16982 & 0.04437 & DOWN \\
\hline CENPW & 1.0746 & 5.7700 & -2.42478 & 0.19064 & DOWN \\
\hline HSD3B1 & 1.2478 & 14.4836 & -3.53701 & 0.02839 & DOWN \\
\hline HSD3B2 & 1.5398 & 6.4124 & -2.05816 & 0.10677 & DOWN \\
\hline SAMD13 & 1.0206 & 5.9732 & -2.54906 & 0.05329 & DOWN \\
\hline NEU4 & 2.6974 & 12.5380 & -2.21666 & 0.05609 & DOWN \\
\hline NPY4R & 1.3809 & 13.3205 & -3.26999 & 0.17219 & DOWN \\
\hline COL11A2 & 1.9754 & 9.7104 & -2.29740 & 0.05077 & DOWN \\
\hline PRRT1 & 2.1848 & 15.7600 & -2.85073 & 0.00387 & DOWN \\
\hline SP5 & 1.5658 & 8.3764 & -2.41948 & 0.17049 & DOWN \\
\hline CSHL1 & 1.4613 & 9.1288 & -2.64318 & 0.02839 & DOWN \\
\hline KRTAP5-10 & 1.3248 & 9.4194 & -2.82982 & 0.19671 & DOWN \\
\hline TRIM31 & 1.2338 & 14.0165 & -3.50599 & 0.00387 & DOWN \\
\hline C5orf60 & 1.4031 & 8.3857 & -2.57932 & 0.01847 & DOWN \\
\hline PSG5 & 1.2016 & 10.6864 & -3.15273 & 0.00387 & DOWN \\
\hline$\angle R R C 10 B$ & 1.1943 & 5.1378 & -2.10497 & 0.04585 & DOWN \\
\hline PCDHGA1 & 2.3688 & 9.5591 & -2.01270 & 0.18429 & DOWN \\
\hline PCDHA5 & 2.3824 & 10.1467 & -2.09054 & 0.14461 & DOWN \\
\hline PCDHA2 & 1.4505 & 9.3862 & -2.69404 & 0.08011 & DOWN \\
\hline PRSS1 & 1.5583 & 14.9059 & -3.25788 & 0.08666 & DOWN \\
\hline CCNI2 & 3.1212 & 17.4700 & -2.48472 & 0.15362 & DOWN \\
\hline MUC12 & 1.0936 & 7.3007 & -2.73891 & 0.01081 & DOWN \\
\hline KRT6A & 2.1158 & 16.2543 & -2.94154 & 0.05024 & DOWN \\
\hline SERPINB5 & 1.1846 & 6.8852 & -2.53913 & 0.04829 & DOWN \\
\hline ZDHHC11B & 1.7757 & 8.0244 & -2.17598 & 0.19291 & DOWN \\
\hline C3orf72 & 1.1782 & 9.5382 & -3.01717 & 0.06906 & DOWN \\
\hline TMEM200C & 1.8587 & 9.5679 & -2.36389 & 0.05077 & DOWN \\
\hline VGLL3 & 1.9752 & 12.0393 & -2.60767 & 0.02537 & DOWN \\
\hline PRSS50 & 1.3241 & 7.1207 & -2.42702 & 0.17431 & DOWN \\
\hline DIO2 & 3.0417 & 13.2519 & -2.12323 & 0.01399 & DOWN \\
\hline C17orf51 & 2.9122 & 17.4716 & -2.58485 & 0.00387 & DOWN \\
\hline KRTAP3-1 & 2.0845 & 19.4546 & -3.22237 & 0.13853 & DOWN \\
\hline$C C D C 19$ & 2.2564 & 12.8781 & -2.51282 & 0.13040 & DOWN \\
\hline ASIC3 & 2.6110 & 11.3970 & -2.12596 & 0.05924 & DOWN \\
\hline OR2F1 & 1.2892 & 16.6556 & -3.69150 & 0.18441 & DOWN \\
\hline ATP6V1G2 & 1.5561 & 11.6176 & -2.90027 & 0.16106 & DOWN \\
\hline PPM1N & 1.3000 & 5.8772 & -2.17663 & 0.08535 & DOWN \\
\hline$P R C D$ & 3.0234 & 16.0721 & -2.41032 & 0.01241 & DOWN \\
\hline IQCJ & 1.4319 & 14.8175 & -3.37128 & 0.03573 & DOWN \\
\hline NUTM2D & 1.4907 & 9.4296 & -2.66124 & 0.05144 & DOWN \\
\hline TBC1D26 & 1.9726 & 8.9793 & -2.18653 & 0.12927 & DOWN \\
\hline AP000974.1 & 1.7410 & 7.0348 & -2.01463 & 0.06125 & DOWN \\
\hline$H M X 1$ & 1.0239 & 7.6265 & -2.89695 & 0.13796 & DOWN \\
\hline GCGR & 4.7686 & 26.2124 & -2.45861 & 0.15536 & DOWN \\
\hline ТТС34 & 1.8101 & 8.8655 & -2.29216 & 0.08263 & DOWN \\
\hline HTR5A-AS1 & 1.3346 & 7.7682 & -2.54117 & 0.19689 & DOWN \\
\hline TAS2R41 & 1.4647 & 7.8757 & -2.42677 & 0.19687 & DOWN \\
\hline C1orf229 & 1.2856 & 5.8539 & -2.18702 & 0.03573 & DOWN \\
\hline$A D R A 2 B$ & 1.9165 & 7.9237 & -2.04774 & 0.04013 & DOWN \\
\hline REX01L11P & 9.6644 & 84.6481 & -3.13073 & 0.00387 & DOWN \\
\hline CKMT1A & 1.0104 & 8.6133 & -3.09166 & 0.10296 & DOWN \\
\hline C5orf66 & 2.3976 & 17.2659 & -2.84825 & 0.01560 & DOWN \\
\hline PRRT4 & 1.2766 & 8.6891 & -2.76688 & 0.03797 & DOWN \\
\hline ZNF469 & 1.2850 & 8.1171 & -2.65915 & 0.00387 & DOWN \\
\hline AC104809.3 & 1.9570 & 13.4865 & -2.78483 & 0.02439 & DOWN \\
\hline OR2T7 & 3.5054 & 20.6900 & -2.56129 & 0.01847 & DOWN \\
\hline CTB-58E17.5 & 1.1962 & 9.1993 & -2.94303 & 0.15873 & DOWN \\
\hline C14orf144 & 1.6183 & 8.4079 & -2.37729 & 0.19041 & DOWN \\
\hline CASP16 & 1.1202 & 6.2886 & -2.48896 & 0.11921 & DOWN \\
\hline C1orf233 & 1.1972 & 5.6246 & -2.23214 & 0.03797 & DOWN \\
\hline PROB1 & 1.7245 & 7.5133 & -2.12330 & 0.02336 & DOWN \\
\hline SFTA3 & 1.4605 & 14.1962 & -3.28101 & 0.00387 & DOWN \\
\hline IQCF3 & 2.7249 & 17.7870 & -2.70653 & 0.05144 & DOWN \\
\hline
\end{tabular}

Table S4 (continued) 


\begin{tabular}{|c|c|c|c|c|c|}
\hline Gene name & Cancer_FPKM & Non-cancer_FPKM & log2(fold change) & FDR & Trend \\
\hline CYP21A2 & 3.0739 & 14.3148 & -2.21935 & 0.14817 & DOWN \\
\hline PSG1 & 1.9864 & 13.4196 & -2.75609 & 0.00880 & DOWN \\
\hline TMEM253 & 1.2096 & 9.3437 & -2.94946 & 0.09524 & DOWN \\
\hline TMEM229A & 1.0733 & 6.8050 & -2.66450 & 0.18519 & DOWN \\
\hline C5orf58 & 1.8844 & 12.7191 & -2.75481 & 0.12011 & DOWN \\
\hline WASH4P & 2.9614 & 12.5209 & -2.08000 & 0.01399 & DOWN \\
\hline FАM203A & 1.5045 & 8.3537 & -2.47317 & 0.12960 & DOWN \\
\hline C8orf56 & 2.1215 & 11.9896 & -2.49862 & 0.16441 & DOWN \\
\hline PATE4 & 2.1381 & 21.3311 & -3.31856 & 0.05385 & DOWN \\
\hline OR2W3 & 2.5016 & 13.9134 & -2.47553 & 0.16472 & DOWN \\
\hline GATSL3 & 6.0146 & 35.4995 & -2.56127 & 0.01560 & DOWN \\
\hline C2orf61 & 1.1734 & 8.0924 & -2.78585 & 0.09193 & DOWN \\
\hline LCE1F & 1.1307 & 11.8595 & -3.39080 & 0.08718 & DOWN \\
\hline TNFRSF13B & 2.4431 & 18.2838 & -2.90380 & 0.02439 & DOWN \\
\hline TDGF1 & 2.1974 & 15.1549 & -2.78591 & 0.01081 & DOWN \\
\hline CDRT1 & 1.8032 & 9.7092 & -2.42882 & 0.09506 & DOWN \\
\hline ARHGDIG & 1.5602 & 8.1089 & -2.37775 & 0.14885 & DOWN \\
\hline$E R V W-1$ & 1.2929 & 12.4272 & -3.26487 & 0.02756 & DOWN \\
\hline PSG11 & 1.3175 & 18.9324 & -3.84499 & 0.00387 & DOWN \\
\hline PSG4 & 1.9314 & 24.3538 & -3.65642 & 0.00387 & DOWN \\
\hline PTCHD4 & 2.1240 & 8.5641 & -2.01151 & 0.03336 & DOWN \\
\hline$R P 11-766 F 14.2$ & 1.0138 & 5.9778 & -2.55987 & 0.01712 & DOWN \\
\hline ATPGV1E2 & 2.7996 & 11.2994 & -2.01296 & 0.12859 & DOWN \\
\hline LINC00923 & 3.0587 & 14.9941 & -2.29340 & 0.18381 & DOWN \\
\hline$R P 11-1102 P 16.1$ & 1.1963 & 5.1457 & -2.10474 & 0.16026 & DOWN \\
\hline EIF5AL1 & 1.1979 & 5.4828 & -2.19446 & 0.02439 & DOWN \\
\hline NOX5 & 1.1329 & 7.2134 & -2.67067 & 0.03135 & DOWN \\
\hline PCDHA3 & 1.9929 & 11.4911 & -2.52756 & 0.15642 & DOWN \\
\hline C21orf119 & 3.3372 & 14.7841 & -2.14733 & 0.03431 & DOWN \\
\hline SALL3 & 2.2937 & 9.7002 & -2.08031 & 0.04187 & DOWN \\
\hline OVCH1-AS1 & 1.4554 & 8.8108 & -2.59784 & 0.13358 & DOWN \\
\hline$R P 11-347 C 12.3$ & 1.2235 & 5.3860 & -2.13824 & 0.14086 & DOWN \\
\hline CLEC5A & 2.0959 & 15.3091 & -2.86873 & 0.08324 & DOWN \\
\hline$R P 11-204 N 11.1$ & 1.9637 & 16.8874 & -3.10433 & 0.14998 & DOWN \\
\hline C16orf95 & 2.8051 & 14.2367 & -2.34350 & 0.07203 & DOWN \\
\hline$R P 11-77 K 12.1$ & 1.0649 & 8.2597 & -2.95544 & 0.19643 & DOWN \\
\hline UBBP4 & 1.3371 & 7.1485 & -2.41857 & 0.09193 & DOWN \\
\hline PTGES3L & 1.0802 & 4.4050 & -2.02789 & 0.12960 & DOWN \\
\hline AC004466.1 & 2.4625 & 13.5172 & -2.45659 & 0.18831 & DOWN \\
\hline AC093677.1 & 1.3382 & 21.4097 & -3.99991 & 0.12182 & DOWN \\
\hline AC010327.2 & 1.0732 & 8.6130 & -3.00460 & 0.14266 & DOWN \\
\hline KRBOX1 & 1.8851 & 9.3947 & -2.31723 & 0.12317 & DOWN \\
\hline
\end{tabular}

